Journal of Computer Science 2 (9): 716-734, 2006

ISSN 1549-3636

(C) 2006 Science Publications,

\title{
Tolerances Applied in Combinatorial Optimization
}

\author{
${ }^{1,2}$ Boris Goldengorin, ${ }^{3}$ Gerold Jäger and ${ }^{3}$ Paul Molitor \\ ${ }^{1}$ Faculty of Economic Sciences, University of Groningen, 9700 AV Groningen, The Netherlands \\ ${ }^{2}$ Department of Applied Mathematics, Khmelnitsky National University, Ukraine \\ ${ }^{3}$ University of Halle-Wittenberg, Computer Science Institute, D-06099 Halle (Saale), Germany
}

\begin{abstract}
In this paper we deal with sensitivity analysis of combinatorial optimization problems and its fundamental term, the tolerance. For three classes of objective functions $(\Sigma, \Pi$, MAX $)$ we give some basic properties on upper and lower tolerances. We show that the upper tolerance of an element is well defined, how to compute the upper tolerance of an element and give equivalent formulations when the upper tolerance is $+\infty$ or $>0$. Analogous results are given for the lower tolerance and some results on the relationship between lower and upper tolerances are given.
\end{abstract}

Key words: Sensitivity analysis, upper tolerance, lower tolerance

\section{INTRODUCTION}

After an optimal solution to a combinatorial optimization problem has been determined, a natural next step is to apply sensitivity analysis ${ }^{[1]}$, sometimes also referred to as post-optimality analysis or what-if analysis $^{[2]}$. Sensitivity analysis is also a wellestablished topic in linear programming ${ }^{[3]}$ and mixed integer programming ${ }^{[2]}$. The purpose of sensitivity analysis is to determine how the optimality of the given optimal solution depends on the input data. There are several reasons for performing sensitivity analysis. In many cases the data used are inexact or uncertain. In such cases sensitivity analysis is necessary to determine the credibility of the optimal solution and conclusions based on that solution. Another reason for performing sensitivity analysis is that sometimes rather significant considerations have not been built into the model due to the difficulty of formulating them. Having solved the simplified model, the decision maker wants to know how well the optimal solution fits in with the other considerations.

The most interesting topic of sensitivity analysis is the special case when the value of a single element in the optimal solution is subject to change. The goal of such perturbations is to determine the tolerances being defined as the maximum changes of a given individual cost (weight, distance, time etc.) preserving the optimality of the given optimal solution. The first successful implicit application of upper tolerances for improving the Transportation Simplex Algorithm is appeared in the so called Vogel's Approximation Method $^{[4]}$ and has been used for a straightforward enumeration of the $k$-best solutions for some positive integer $k^{[5,6]}$ as well as a base of the MAX-REGRET heuristic for solving the three-index assignment problem $^{[7]}$.
The values of upper tolerances have been applied for improving the computational efficiency of heuristics and branch-and-bound algorithms for solving different classes of NP-hard problems (for example of the traveling salesman problem (TSP) ${ }^{[8-11]}$ ). Also for the TSP, Helsgaun ${ }^{[12]}$ improved the Lin-Kernighan heuristic by using the lower tolerances to the minimum 1 -tree with great success.

Computational issues of tolerances to the minimum spanning tree problem and TSP are addressed in Chin and Hock $^{[13]}$, Gordeev et al..$^{[14]}$, Gusfield ${ }^{[15]}$, Kravchenko et al. ${ }^{[16]}$, Libura ${ }^{[17]}$, Ramaswamy and Chakravarti $^{[18]}$, Shier and Witzgall ${ }^{[19]}$, Sotskov ${ }^{[20]}$, $\operatorname{Tarjan}^{[21]}$. Recently, Volgenant ${ }^{[22]}$ has suggested an $O\left(n^{3}\right)$ algorithm for computing the upper and lower tolerances for all arcs in the Assignment Problem. Ramaswamy et al. have reviewed the sensitivity analysis problem for the maximum capacity path problem $\left({ }^{[23]}\right.$ and references within) and suggested an elegant reduction of the sensitivity analysis problem for the shortest path and maximum capacity path problems in an undirected network to the minimum cost interval problem. For an extensive account on computational issues of upper and lower tolerances in the context of sensitivity analysis in combinatorial optimization ${ }^{[2,3,24,25,26]}$.

The purpose of this paper is to give an overview over the terms of upper and lower tolerances for the three most natural types $\Sigma$, П, MAX of objective functions. To our best knowledge we have not found any publications treating the sensitivity analysis problem for a general class of combinatorial optimization problems with different types of objective functions. The paper is the first which deals with tolerances in an exact, general and comprehensive way, so that discrepancies of previous descriptions can be

Corresponding Author: $\quad$ Boris Goldengorin, Faculty of Economic Sciences, University of Groningen, 9700 AV Groningen, The Netherlands 
avoided, e.g. all of above mentioned papers have used but not indicated an important assumption that the set of feasible solutions to a combinatorial optimization problem under consideration is independent of the cost (objective) function. Furthermore, this coherent consideration leads to new results about tolerances.

The paper is organized as follows. In section 2 we define a combinatorial minimization problem and give all notations which are necessary for the terms of upper and lower tolerances. In section 3 we define the upper tolerance and give characteristics of it. Especially, we show that the upper tolerance is well defined with respect to the problem instance, i.e., that the upper tolerance of an element with respect to an optimal solution $S^{\star}$ of a problem instance $P$ doesn't depend on $S^{\star}$ but only on $P$ itself. Furthermore we show how to characterize elements with upper tolerance $+\infty$ or $>0$ and how the upper tolerance can be computed. In section 4 we show similar relations for the lower tolerance. In section 5 we give relationships between lower and upper tolerances which mostly are direct conclusions from the sections 3 and 4. Our main result for objective functions of type $\sum$ is that under certain conditions the minimum value of upper tolerance equals the minimum value of lower tolerance and the maximum value of upper tolerance equals the maximum value of lower tolerance. Similar results for objective functions of type $\Pi$, MAX do not hold. The non-trivial proofs of the statements can be found in section 6. We summarize our paper in section 7 and propose directions for future research.

A preliminary version of this paper appeared in the proceedings of the 2nd International Conference on Algorithmic Aspects in Information and Management (AAIM), Hong Kong, China, June 20-22, 2006, Lecture Notes in Comput. Sci., 4041: 194-206.

\section{COMBINATORIAL MINIMIZATION PROBLEMS}

A combinatorial minimization problem $P$ is given by a tuple $\left(E, D, c, f_{c}\right)$ with

* $\quad E$ is a finite ground set of elements.

* $\quad D \subseteq 2^{E}$ is the set of the feasible solutions.

* $\quad c: E \rightarrow \mathbf{R}$ is the function which assigns costs to each single element of $E$.

* $f_{c}: 2^{E} \rightarrow \mathbf{R}$ is the objective (cost) function which depends on function $c$ and assigns costs to each subset of $E$.

A subset $S^{\star} \subseteq E$ is called an optimal solution of $P$, if $S^{\star}$ is a feasible solution and the costs $f_{c}\left(S^{\star}\right)$ of $S^{\star}$ are minimal (analogous considerations can be made if the costs have to be maximized, i.e., for combinatorial maximization problems), i.e., $S^{\star} \in D$ and $f_{c}\left(S^{\star}\right)=\min \left\{f_{c}(S) ; S \in D\right\}$. We denote the set of optimal solutions by $D^{\star}$. There are some particular monotone cost functions which often occur in practice:

* [Type $\Sigma$ ] The cost function $f_{c}: 2^{E} \rightarrow \mathbf{R}$ is of type $\sum$, if for each $S \in 2^{E}: f_{c}(S)=\sum_{\bar{e} \in S} c(\bar{e})$ holds.

* [Type $\Pi]$ The cost function $f_{c}: 2^{E} \rightarrow \mathbf{R}$ is of type $\Pi$, if for each $S \in 2^{E}: f_{c}(S)=\prod_{\bar{e} \in S} c(\bar{e})$ and for each $e \in E: c(e)>0$ holds.

* $\quad$ [Type MAX] The cost function $f_{c}: 2^{E} \rightarrow \mathbf{R}$ is of type MAX (such a cost function is also called bottleneck function), if for each $S \in 2^{E}$ : $f_{c}(S)=\max \{c(\bar{e}) ; \bar{e} \in S\}$ holds.

These three objective functions are monotone, i.e., the costs of a subset of $E$ don't become cheaper if the costs of a single element of $E$ are increased.

In the remainder of the paper, we only consider combinatorial minimization problems $P=\left(E, D, c, f_{c}\right)$ which fulfill the following three conditions.

Condition 1: The set $D$ of the feasible solutions of $P$ is independent of function $c$.

Condition 2: The cost function $f_{c}: 2^{E} \rightarrow \mathbf{R}$ is either of type $\sum$, type $\Pi$, or type MAX.

Condition 3: There is at least one optimal solution of $P$, i.e., $D^{\star} \neq \varnothing$.

Note that the Traveling Salesman Problem (TSP), Minimum Spanning Tree (MST) and many other combinatorial minimization problems fulfill these three conditions $^{[27]}$.

Given a combinatorial minimization problem $P=\left(E, D, c, f_{c}\right)$, we obtain a new combinatorial minimization problem if we increase the costs of a single element $e \in E$ by some constant $\alpha \in \mathbf{R}$. We will denote the new problem by $P_{\alpha, e}=\left(E, D, c_{\alpha, e}, f_{c_{\alpha, e}}\right)$, which is formally defined by $c_{\alpha, e}(\bar{e})=\left\{\begin{array}{cl}c(\bar{e}) & , \text { if } \bar{e} \neq e \\ c(\bar{e})+\alpha & , \text { if } \bar{e}=e\end{array}\right.$ for each $\bar{e} \in E$ and $f_{c_{\alpha, e}}$ is of the same type as $f_{c}$. Further define $P_{-\infty, e}=\lim _{\alpha \rightarrow-\infty} P_{\alpha, e}$ and $P_{+\infty, e}=\lim _{\alpha \rightarrow+\infty} P_{\alpha, e}$.

We need some more notations with respect to a combinatorial minimization problem $P$. Let $e$ be a single element of $E$.

* $f_{c}(P)$ denotes the costs of an optimal solution $S^{\star}$ of $P$. 
* For $M \subseteq D, f_{c}(M)$ denotes the costs of the best solution included in $M$. The costs $f_{c}(S)$ of either infeasible or empty set $S$ are defined as $+\infty$. Obviously, for each $M \subseteq D: f_{c}(P) \leq f_{c}(M)$ holds.

* $\quad D_{-}(e)$ denotes the set of the feasible solutions of $D$ each of which doesn't contain the element $e \in E$, i.e., $D_{-}(e)=\{S \in D ; e \notin S\}$. Analogously, $D_{+}(e)$ denotes the set of the feasible solutions $D$ each of which contains the element $e \in E$, i.e., $D_{+}(e)=\{S \in D ; e \in S\}$.

* $\quad D_{-}^{\star}(e)$ denotes the set of the best feasible solutions of $D$ each of which doesn't contain the element $e \in E$, i.e.,

$D_{-}^{\star}(e)$

$=\left\{S \in D ; e \notin S\right.$ and $\left(\forall S^{\prime} \in D\right)\left(e \notin S^{\prime} \Rightarrow f_{c}(S) \leq f_{c}\left(S^{\prime}\right)\right\}$

The elements of $D_{-}^{\star}(e)$ are called $S_{-}^{\star}(e)$.

Analogously, $D_{+}^{\star}(e)$ denotes the set of the best feasible solutions $D$ each of which contains the element $e \in E$, i.e.,

$D_{+}^{\star}(e)$

$=\left\{S \in D ; e \in S\right.$ and $\left(\forall S^{\prime} \in D\right)\left(e \in S^{\prime} \Rightarrow f_{c}(S) \leq f_{c}\left(S^{\prime}\right)\right\}$

The elements of $D_{+}^{\star}(e)$ are called $S_{+}^{\star}(e)$.

\section{UPPER TOLERANCES}

Let $P=\left(E, D, c, f_{c}\right)$ be a combinatorial minimization problem which fulfills Conditions 1,2 and 3. Consider an optimal solution $S^{\star}$ of $P$ and fix it.

For a single element $e$ of this optimal solution $S^{\star}$, let the upper tolerance $u_{S^{\star}}(e)$ of element $e$ with respect to $S^{\star}$ be the supremum by which the costs of $e$ can be increased such that $S^{\star}$ remains an optimal solution, provided that the costs of all other elements $\bar{e} \in E \backslash\{e\}$ remain unchanged, i.e., for each $e \in S^{*}$ the upper tolerance is defined as follows:

$u_{S^{\star}}(e):=\sup \left\{\alpha \in \mathbf{R} ; S^{\star}\right.$ is an optimal solution of $\left.P_{\alpha, e}\right\}$

Because of the monotonicity of the cost function it holds:

$u_{S^{\star}}(e):=\inf \left\{\alpha \in \mathbf{R} ; S^{\star}\right.$ is not an optimal solution of

$$
\left.P_{\alpha, e}\right\}
$$

As $S^{\star}$ is an optimal solution of $P_{0, e}$, which is $P$, the upper tolerance $u_{S^{\star}}(e)$ is either a non-negative number or $+\infty$. Because of Condition 2, for each $e \in S^{\star}$ with $u_{S^{\star}}(e)<+\infty$, it holds:
$u_{S^{\star}}(e):=\max \left\{\alpha \in \mathbf{R} ; S^{\star}\right.$ is an optimal solution of $\left.P_{\alpha, e}\right\}$

Theorem 1: Let $S^{\star}$ be an optimal solution of $P$ with $e \in S^{*} . e$ is contained in every feasible solution of $P$ if and only if $u_{S^{\star}}(e)=+\infty$, i.e., $e \in \bigcap_{S \in D} S \Leftrightarrow u_{S^{\star}}(e)=+\infty$.

Theorem 2: The upper tolerance of an element doesn't depend on a particular optimal solution of $P$, i.e.,

$\left(\forall S_{1}, S_{2} \in D^{\star}\right)\left(\forall e \in S_{1} \cap S_{2}\right) u_{S_{1}}(e)=u_{S_{2}}(e)$

Thus, if a single element $e \in E$ is contained in at least one optimal solution $S^{\star}$ of $P$, the upper tolerance of $e$ doesn't depend on that particular optimal solution $S^{\star}$ but only on problem $P$ itself. Hence, we can refer to the upper tolerance of $e$ with respect to an optimal solution $S^{\star}$ as upper tolerance of $e$ with respect to $P$, $u_{P}(e)$.

Note that the upper tolerance of an element $e$ which is not contained in any optimal solution is not defined. For these elements $e \in E$, we set $u_{P}(e):=\mathrm{UNDEFINED}$.

Theorem 3: If $e \in E$ with $u_{P}(e) \notin\{$ UNDEFINED, $+\infty\}$, then for all $\varepsilon>0$ the element $e$ is not contained in any optimal solution of $P_{u_{P}(e)+\varepsilon, e}$.

Theorem 3 states that, for all $e \in E$ with $u_{P}(e) \neq \mathrm{UNDEFINED}$ and $u_{P}(e) \neq+\infty$, increasing the costs of $e$ by $u_{P}(e)+\varepsilon$ for $\varepsilon>0$ makes the element uninteresting for optimal solutions.

Theorem 4: For each single element $e \in E$ which is contained in at least one optimal solution $S^{\star}$ of $P$, the upper tolerance of $e$ is given by:

* $u_{P}(e)=f_{c}\left(D_{-}^{\star}(e)\right)-f_{c}(P)$, if the cost function is of type $\sum$

* $\quad u_{P}(e)=\frac{f_{c}\left(D_{-}^{\star}(e)\right)-f_{c}(P)}{f_{c}(P)} \cdot c(e)$, if the cost function is of type $\Pi$

* $\quad u_{P}(e)=f_{c}\left(D_{-}^{\star}(e)\right)-c(e)$, if the cost function is of type MAX

Theorem 5: For each single element $e \in E$ it holds for a cost function of type $\sum, \Pi$ and MAX : $f_{c}\left(D_{-}^{\star}(e)\right)=f_{c_{+\infty, e}}(P)$.

Theorem 4 and Theorem 5 tell us how to compute the upper tolerance of a single element $e \in E$ with respect to $P$. We observe ${ }^{[18,28]}$ :

Corollary 1: The upper tolerance of one element $e \in E$ can be computed by solving two different instances of 
$P$ for a cost function of type $\Sigma, \Pi$ and solving one instance of $P$ for a cost function of type MAX, i.e., the computation of the upper tolerance has the same complexity as $P$ itself.

Theorem 6: If the cost function is either of type $\Sigma$ or $\Pi$, then a single element $e$ in at least one optimal solution is contained in every optimal solution if and only if its upper tolerance is greater than 0 , i.e., $e \in \bigcap_{S^{\star} \in D^{\star}} S^{\star} \Leftrightarrow u_{P}(e)>0 \quad$ or $\quad$ equivalently $\bigcap_{S^{\star} \in D^{\star}} S^{\star}=\left\{e ; u_{P}(e)>0\right\}$.

Theorem 6 characterizes those elements which are contained in every optimal solution. We only have to know the upper tolerance of an element. Unfortunately, this property doesn't hold for a cost function of type MAX .

Remark 1: In general, for a cost function of type MAX only the direction " $\Rightarrow$ " of Theorem 6 holds, but not the direction " $\Leftarrow$ ".

Corollary 2: Let the cost function be either of type $\Sigma$ or of type $\Pi$. There is only one optimal solution of $P$ if and only if $u_{P}(e)>0$ for all $e$ with $u_{P}(e) \neq \mathrm{UNDEFINED}$.

Remark 2: Note that Condition 1 is crucial for all these properties, in particular for Theorem 4.

\section{LOWER TOLERANCES}

Now, let $S^{\star}$ be an optimal solution of $P$ which doesn't contain the element $e \in E$. Analogously to the considerations which we have made with respect to the upper tolerance, we can ask for the supremum by which the costs of element $e$ can be decreased such that $S^{\star}$ remains an optimal solution, provided that the costs of all other elements remain unchanged. More formally, we define for all $e \in E \backslash S^{\star}$ :

$l_{S^{\star}}(e):=\sup \left\{\alpha \in \mathbf{R} ; f_{c_{-\alpha, e}}\right.$ is monotone and

$$
\left.S^{\star} \text { is an optimal solution of } P_{-\alpha, e}\right\}
$$

Because of the monotonicity of the cost function it holds:

$$
\begin{aligned}
& l_{S^{\star}}(e):=\inf \left\{\alpha \in \mathbf{R} ; f_{c_{-\alpha, e}}\right. \text { is monotone and } \\
& \left.\qquad S^{\star} \text { is not an optimal solution of } P_{-\alpha, e}\right\}
\end{aligned}
$$

Note that if the cost function of the combinatorial minimization problem is of type $\Pi$, the costs of the elements have to be greater than zero to guarantee monotonicity. In the following, let $\delta_{\max }(e)$ be defined as $\delta_{\max }(e):= \begin{cases}+\infty & \text {,if } f_{c} \text { is either of type } \sum \text { or } \\ c(e) & \text { of type MAX } \\ \text {,if } f_{c} \text { is of type } \Pi\end{cases}$

$\delta_{\max }(e)$ is the supremum by which element $e$ can be decreased such that the cost function remains either of type $\Sigma, \Pi$, or MAX .

As $S^{\star}$ is an optimal solution of $P_{-0, e}$ which is $P$, the lower tolerance $l_{S^{*}}(e)$ is either a non-negative number or $+\infty$ if $e \notin S^{\star}$. More exactly, it holds for each $e \in E \backslash S^{\star}: \quad 0 \leq l_{S^{\star}}(e) \leq \delta_{\max }(e)$

Because of Condition 2, for each $e \in E \backslash S^{\star}$ and each $l_{S^{\star}}(e)<\delta_{\max }(e)$, it holds:

$l_{S^{\star}}(e)=\max \left\{\alpha \in \mathbf{R} ; f_{c_{-\alpha, e}}\right.$ is monotone and

$$
\left.S^{\star} \text { is an optimal solution of } P_{-\alpha, e}\right\}
$$

Theorem 7: Let the cost function be of type $\sum$ or $\Pi$ and let $S^{\star}$ be an optimal solution of $P$. Then, an element $e$ isn't contained in a feasible solution if and only if $\quad l_{S^{\star}}(e)=\delta_{\max }(e), \quad$ i.e., $e \in E \backslash \bigcup_{S \in D} S \Leftrightarrow l_{S^{\star}}(e)=\delta_{\max }(e)$.

Remark 3: In general, for a cost function of type MAX only the direction " $\Rightarrow$ " of Theorem 7 holds, but not the direction " $\Leftarrow$ ".

Remark 3 partly puts lower tolerances with respect to a cost function of type MAX in question. It states that the lower tolerance of an element can be very large, namely $+\infty$, although this element can be included in a feasible solution. It can be shown that the element can be included in an optimal solution. This contradicts the intuition that an element with large lower tolerance is not a "good" element and should not be included in solutions by heuristics.

Theorem 8: The lower tolerance of an element doesn't depend on a particular optimal solution of $P$, i.e., $\left(\forall S_{1}, S_{2} \in D^{\star}\right)\left(\forall e \notin S_{1} \cup S_{2}\right): l_{S_{1}}(e)=l_{S_{2}}(e)$.

Thus, if there is at least one optimal solution $S^{\star}$ of $P$ which doesn't contain element $e$, the lower tolerance of $e$ doesn't depend on that particular optimal solution but only on problem $P$ itself. As for upper tolerances, we can refer to the lower tolerance of $e$ with respect to an optimal solution $S^{\star}$ as lower tolerance of $e$ with respect to $P, l_{P}(e)$.

The lower tolerance of an element $e$ which is contained in every optimal solution is not defined, yet. For these elements $e$, we set $l_{p}(e):=\mathrm{UNDEFINED}$. 
Theorem 9: If $e \in E$ is a single element with $l_{P}(e) \notin\left\{\mathrm{UNDEFINED}, \delta_{\max }(e)\right\}$, then element $e$ is contained in every optimal solution of $P_{-\left(l_{p}(e)+\varepsilon\right), e}$ for all $0<\varepsilon<\delta_{\max }(e)-l_{P}(e)$.

Theorem 9 states that if we decrease the costs of $e$ by more than $l_{P}(e)$, then an optimal solution will contain element $e$, provided that $l_{P}(e)$ is neither UNDEFINED nor $\delta_{\max }(e)$.

Let for a single element $e \in E$ and a cost function of type MAX

$g(e):= \begin{cases}\min _{S \in D_{+}(e)} \max _{a \in S \backslash(e\}}\{c(a)\} & \text {,if } D_{+}(e) \neq \varnothing \\ +\infty & \text {,if } D_{+}(e)=\varnothing\end{cases}$

Obviously, it holds:

$f_{c_{-\infty, e}}(P)=\min \left\{g(e), f_{c}\left(D_{-}^{\star}(e)\right)\right\}$

Theorem 10: For each single element $e \in E$ it holds:

* $f_{c}\left(D_{+}^{\star}(e)\right)=\lim _{K \rightarrow+\infty}\left(f_{c_{-K, e}}(P)+K\right)$, if the cost function is of type $\Sigma$

* $f_{c}\left(D_{+}^{\star}(e)\right)=\lim _{K \rightarrow c(e)^{-}}\left(\frac{f_{c-e_{c}}(P)}{c(e)-K} \cdot c(e)\right)$, if the cost function is of type $\Pi$

* $f_{c}\left(D_{+}^{\star}(e)\right)=\max \{g(e), c(e)\}$, if the cost function is of type MAX

Theorem 11: For each single element $e \in E$ with $l_{P}(e) \neq \mathrm{UNDEFINED}$, the lower tolerance of $e$ with respect to $P$ is given by:

* $\quad l_{P}(e)=f_{c}\left(D_{+}^{\star}(e)\right)-f_{c}(P)$, if the cost function is of type $\Sigma$

* $\quad l_{P}(e)=\frac{f_{c}\left(D_{+}^{\star}(e)\right)-f_{c}(P)}{f_{c}\left(D_{+}^{\star}(e)\right)} \cdot c(e)$, if the cost function is of type $\Pi$

$* \quad l_{P}(e)=\left\{\begin{array}{ll}c(e)-f_{c}(P) & , \text { if } g(e)<f_{c}(P) \\ +\infty & , \text { otherwise }\end{array}\right.$, if the cost function is of type MAX

Theorem 10 and Theorem 11 tell us how to compute the lower tolerance of a single element $e \in E$ with respect to $P$. We observe

Corollary 3: The lower tolerance of a single element $e \in E$ can be computed by solving two different instances of $P$ for a cost function of type $\Sigma, \Pi$ and solving one instance of $P$ for a cost function of type MAX, i.e., the computation of the lower tolerance has the same complexity as $P$ itself.

Theorem 12: If the cost function is either of type $\sum$ or $\Pi$, then a single element $e \in E$ isn't contained in any optimal solution if and only if its lower tolerance is greater than 0 , i.e., $e \notin \bigcup_{S^{\star} \in D^{\star}} S^{\star} \Leftrightarrow l_{P}(e)>0 \quad$ or equivalently $E \backslash \bigcup_{S^{\star} \in D^{\star}} S^{\star}=\left\{e ; l_{P}(e)>0\right\}$. Theorem 12 characterizes those elements which are never included in an optimal solution.

Remark 4: In general, for a cost function of type MAX only the direction " $\Rightarrow$ " of Theorem 12 holds, but not the direction " $\Leftarrow$ ".

\section{RELATIONSHIP BETWEEN LOWER AND UPPER TOLERANCES}

The following properties hold for each cost function $f_{c}$ either of type $\Sigma$ or $\Pi$.

Corollary 4: Let the cost function be either of type $\Sigma$ or of type $\Pi$. For all $e \in E$, the equivalence $l_{P}(e)=\mathrm{UNDEFINED} \Leftrightarrow u_{P}(e)>0$ holds.

Proof: The statement follows from Theorem 6 and the definition of lower tolerance.

Corollary 5: Let the cost function be either of type $\Sigma$ or of type $\Pi$. For all $e \in E$, the equivalence $u_{P}(e)=\operatorname{UNDEFINED} \Leftrightarrow l_{P}(e)>0$ holds.

Proof: The statement follows from Theorem 12 and the definition of upper tolerance.

Corollary 6: Let the cost function be either of type $\Sigma$ or of type $\Pi$. For each $e \in E$ which is contained in at least one optimal solution of $P$ but not in all, i.e., $e \in \bigcup_{S^{\star} \in D^{\star}} S^{\star} \quad$ and $e \notin \bigcap_{S^{\star} \in D^{\star}} S^{\star}$, the equation $u_{P}(e)=l_{P}(e)=0$ holds.

Proof: Both the upper tolerance and the lower tolerance of $e$ are defined. $u_{P}(e)=0$ holds because of Theorem 6. $l_{P}(e)=0$ holds because of Theorem 12 .

Actually, there are much more close interrelations between lower and upper tolerances.

Let $u_{P, \min }=\min \left\{\quad u_{P}(e) ; \quad e \in E \quad\right.$ and $\quad u_{P}(e) \neq$ UNDEFINED $\}$ and $l_{P, \text { min }}=\min \left\{\quad l_{P}(e) ; \quad e \in E\right.$ and $\left.l_{P}(e) \neq \mathrm{UNDEFINED}\right\}$ be the smallest upper and lower tolerance with respect to $P$. Furthermore, let $\Delta_{P, \min }$ be defined as $\Delta_{P, \min }=\min \left\{\delta_{\max }(e) ; e \in E\right\}$. 
Corollary 7: Let the cost function be either of type $\Sigma$ or of type $\Pi$. Provided that there are at least two different optimal solutions, i.e., $\left|D^{\star}\right| \geq 2$, the equation $u_{P, \min }=l_{P, \min }=0$ holds.

Proof: As there are at least two optimal solutions $S_{1}$ and $S_{2}$, there is an element $e_{1}$ with $e_{1} \in S_{1} \backslash S_{2}$ or $e_{1} \in S_{2} \backslash S_{1}$. Thus, $e_{1} \in \bigcup_{S^{\star} \in D^{\star}} S^{\star}$ and $e_{1} \notin \bigcap_{S^{\star} \in D^{\star}} S^{\star}$. By Corollary 6, these two properties of $e_{1}$ imply $u_{P}\left(e_{1}\right)=0$ and $l_{P}\left(e_{1}\right)=0$. Thus, $u_{P, \min }=l_{P, \min }=0$ holds.

Much more interesting is the case that there is only one optimal solution. Here, both the minimal upper tolerance and the minimal lower tolerance are greater than 0 . Nevertheless, they are equal. First, we analyze the special case that there is only one feasible solution of $P$.

Lemma 1: Let the cost function be either of type $\sum$ or of type $\Pi$. If the set $D$ of the feasible solutions of $P$ consists of only one element, say $S$, i.e., $|D|=1$, then $u_{P, \min }=+\infty$ and

$$
\begin{aligned}
& * \quad l_{P, \min }=+\infty \quad \text {,if } S=E \\
& \text { * } l_{P, \min }=\Delta_{P, \min } \quad \text {, if } S=\varnothing \\
& \text { * } l_{P, \text { min }} \geq \Delta_{P, \text { min }} \quad \text {, if } S \neq E \text { and } S \neq \varnothing
\end{aligned}
$$

Remark 5: Note that for the set of the feasible solutions $D$ we have: $D \neq \varnothing$ (Condition 3), but nevertheless it might hold: $\varnothing \in D$.

Corollary 8: Let the cost function be of type $\sum$. If the set $D$ consists of only one element, i.e., $|D|=1$, then $u_{P, \min }=l_{P, \min }=+\infty$ holds.

Proof: The corollary is implied by Lemma 1 as $\Delta_{P, \min }=+\infty$ for a cost function of type $\sum$.

Lemma 2: Let the cost function be of type $\sum$. Provided that no feasible solution is a subset of another feasible solution and there are at least two different feasible solutions but only one optimal solution, i.e., $|D| \geq 2 \quad$ and $\left|D^{\star}\right|=1$, then the equation $u_{P, \text { min }}=l_{P, \text { min }}$ holds. In particular, $0<l_{P, \text { min }} \neq+\infty$ and $0<u_{P, \text { min }} \neq+\infty$.
Theorem 13: Let the cost function be of type $\sum$. Provided that no feasible solution is a subset of another feasible solution, then the equation $u_{P, \min }=l_{P, \min }$ holds.

Proof: The statement is implied by Corollary 7, Corollary 8 and Lemma 2.

Remark 6: If we relax the condition that no feasible solution is a subset of another feasible solution, then Theorem 13 doesn't hold.

Remark 7: In general, Theorem 13 doesn't hold for a cost function of type $\Pi$.

Remark 8: In general, Theorem 13 doesn't hold for a cost function of type MAX.

Corollary 9: Let the cost function be of type $\sum$. Provided that no feasible solution is a subset of another feasible solution, there is only one optimal solution of $P \quad$ if and only if $l_{P}(e)>0$ for all $e$ with $l_{P}(e) \neq$ UNDEFINED .

Proof: The statement follows from Corollary 2, Theorem 13 and the definition of $u_{P, \min }$ and $l_{P, \min }$.

Finally, we consider the largest upper and lower tolerance with respect to $P, u_{P, \max }=\max \left\{u_{P}(e)\right.$; $e \in E$ and $\left.u_{P}(e) \neq \mathrm{UNDEFINED}\right\}$ and $l_{P, \max }=\max \{$ $l_{P}(e) ; e \in E$ and $l_{P}(e) \neq$ UNDEFINED $\}$. We define $G:=\quad\left\{e \in \bigcup_{S^{\star} \in D^{\star}} S^{\star} ; \quad u_{P}(e)=u_{P, \max }\right\} \quad$ and $\quad H:=$ $\left\{e \in E \backslash \bigcap_{S^{\star} \in D^{\star}} S^{\star} ; l_{P}(e)=l_{P, \max }\right\}$.

We call the set of feasible solutions $D$ connected, if $D$ satisfies:
a)

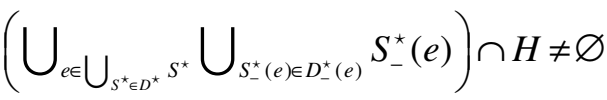
b) $\quad\left(\bigcup_{e \in E \backslash \bigcap_{S^{\star} \in D^{\star}} S^{\star}} \bigcup_{S_{+}^{\star}(e) \in D_{+}^{\star}(e)}\left(E \backslash S_{+}^{\star}(e)\right)\right) \cap G \neq \varnothing$

It is easy to see that conditions a) and b) are equivalent to the conditions a') and b'):
$\left.\mathrm{a}^{\prime}\right) \quad \exists e \in \bigcup_{S^{\star} \in D^{\star}} S^{\star} \exists S_{-}^{\star}(e) \in D_{-}^{\star}(e)$ :
$S_{-}^{\star}(e) \cap H \neq \varnothing$
b') $\exists e \in E \backslash \bigcap_{S^{\star} \in D^{\star}} S^{\star} \exists S_{+}^{\star}(e) \in D_{+}^{\star}(e)$ :
$\left(E \backslash S_{+}^{\star}(e)\right) \cap G \neq \varnothing$ 
Theorem 14: Let the cost function be of type $\sum$. If the set of the feasible solutions $D$ is connected, then the equation $u_{P, \max }=l_{P, \max }$ holds.

We illustrate the conditions a) and b) and Theorem 14 by the following combinatorial minimization problem $P=\left(E, D, c, f_{c}\right)$ :

* $E=\{v, x, y, z\}$ with $c(v)=1, c(x)=2, c(y)=4$ and $c(z)=8$

* $D=\{\{v, x\},\{y, z\}\}$

* $f_{c}$ is a cost function of type $\Sigma$

The only optimal solution is $\{v, x\}$. It holds $u_{P}(v)=9$ and $u_{P}(x)=9$ which implies $u_{P, \max }=9$ and $l_{P}(y)=9$ and $l_{P}(z)=9$ which implies $l_{P, \max }=9$. Therefore $u_{P, \max }=l_{P, \max }$. Furthermore it holds: $G=\{v, x\}, \quad H=\{y, z\}, \quad D_{-}^{\star}(v)=\{\{y, z\}\}$, $D_{-}^{\star}(x)=\{\{y, z\}\}, D_{+}^{\star}(y)=\{\{y, z\}\}$ and $D_{+}^{\star}(z)=\{\{y, z\}\}$. As condition a') and condition b') hold, $D$ is connected.

Remark 9: The condition that the set of the feasible solutions $D$ is connected is only a sufficient, but not a necessary condition for $u_{P, \max }=l_{P, \max }$, i.e., there is a combinatorial minimization problem, where $u_{P, \max }=l_{P, \max }$, although $D$ is not connected.

Remark 10: In general, Theorem 14 doesn't hold for a cost function of type $\Pi$.

Remark 11: In general, Theorem 14 doesn't hold for a cost function of type MAX.

\section{PROOFS}

\section{Proofs of the properties of upper tolerances}

Proof of Theorem 1: For the direction " $\Rightarrow$ " we only have to prove that an optimal solution $S^{\star}$ remains optimal if the costs of an element $e \in S^{*}$ which is contained in every feasible solution are increased. We prove it by case differentiation:

* [The cost function $f_{c}$ is of type $\Sigma$ ]

As element $e$ is included in every feasible solution of $P$, increasing the costs of element $e$ by $\alpha>0$ increases the costs of all feasible solutions of $P$ by the term $\alpha$. Hence, optimal solutions of $P$ are optimal solutions of $P_{\alpha, e}$, too.

* [The cost function $f_{c}$ is of type $\Pi$ ]

As element $e$ is included in every feasible solution of $P$, increasing the costs of element $e$ by $\alpha>0$ increases the costs of all optimal solutions of $P$ by the term $\alpha \cdot \frac{f_{c}(P)}{c(e)}$ and all other feasible solutions $S$ of $P$ by the term $\alpha \cdot \frac{f_{c}(S)}{c(e)}$ which is greater than or equal to $\alpha \cdot \frac{f_{c}(P)}{c(e)}$. Hence, optimal solutions remain optimal.

* [The cost function $f_{c}$ is of type MAX ]

If the costs of element $e$ are increased by $\alpha \leq f_{c}(P)-c(e)$, optimal solutions of $P$ obviously are optimal solutions of $P_{\alpha, e}$, too, because the new costs of $e$ are less than or equal $f_{c}(P)$.

If the costs of element $e$ are increased by $\alpha>f_{c}(P)-c(e)$, the costs of a formerly optimal solution becomes $c(e)+\alpha$ and the costs of each feasible solution are greater than or equal $c(e)+\alpha$. Hence, optimal solutions remain optimal.

To prove the other direction, assume that there is a feasible solution $S \in D$ with $e \notin S$. Increasing the costs of $e$ by some $\gamma>0$ (choose $\gamma$ large enough) results in $f_{c_{\gamma, e}}\left(S^{\star}\right)>f_{c_{\gamma, e}}(S)$ and $S^{\star}$ isn't an optimal solution of $P_{\gamma, e}$. Thus, the upper tolerance $u_{P}(e)$ of $e$ is less than $\gamma$ which is in contradiction to $u_{S^{*}}(e)=+\infty$.

Proof of Theorem 2: The statement follows from Lemma 3, 4 and 5 which we prove in the following.

Lemma 3: (1) holds for a cost function of type $\sum$.

Proof: First, consider the case that $u_{S_{1}}(e)=+\infty$. By Theorem 1, $e \in \bigcap_{S \in D} S$ and thus, $u_{S_{2}}(e)=+\infty$ holds, too.

In the following, we assume that $u_{S_{1}}(e) \neq+\infty$ and $u_{S_{2}}(e) \neq+\infty$.

Let us prove $u_{S_{1}}(e) \geq u_{S_{2}}(e)$, now.

As both solutions $S_{1}$ and $S_{2}$ are optimal, the equation

$\sum_{\bar{e} \in S_{1}} c(\bar{e})=\sum_{\bar{e} \in S_{2}} c(\bar{e})$

holds. Furthermore, the following statements are true:

* By Condition 1 , both $S_{1}$ and $S_{2}$ are feasible solutions of $P_{u_{S_{2}}(e), e}$.

* As $e$ is an element of both $S_{1}$ and $S_{2}$, the costs of $S_{1}$ and $S_{2}$ increase by the term $\alpha$, respectively, if the costs of $e$ are increased by $\alpha$ :

$$
f_{c_{\alpha, e}}\left(S_{1}\right)=\sum_{\bar{e} \in S_{1} \backslash\{e\}} c(\bar{e})+(c(e)+\alpha)
$$




$$
\begin{aligned}
& =\sum_{\bar{e} \in S_{1}} c(\bar{e})+\alpha \\
& =\sum_{\bar{e} \in S_{2}} c(\bar{e})+\alpha \quad \text { see (3) } \\
& =\sum_{\bar{e} \in S_{2} \backslash\{e\}} c(\bar{e})+(c(e)+\alpha)=f_{c_{\alpha, e}}\left(S_{2}\right)
\end{aligned}
$$

This implies that, for all $\alpha>0, S_{1}$ is an optimal solution of $P_{\alpha, e}$ if $S_{2}$ is an optimal solution of $P_{\alpha, e}$. Hence, $u_{S_{1}}(e) \geq u_{S_{2}}(e)$ holds.

Obviously, the relation $u_{S_{1}}(e) \leq u_{S_{2}}(e)$ can be shown analogously.

Lemma 4: (1) holds for a cost function of type $\Pi$.

Proof: The case $u_{S_{1}}(e)=+\infty$ can be shown analogously to the proof of Lemma 3.

In the following, we assume that $u_{S_{1}}(e) \neq+\infty$ and $u_{S_{2}}(e) \neq+\infty$.

The statement of Lemma 4 can be proven analogously to Lemma 3 because of the following two facts:

* For each $\quad e \in S_{1} \cap S_{2}: \quad \prod_{\bar{e} \in S_{1} \backslash\{e\}} c(\bar{e})=$ $\prod_{\left.\bar{e} \in S_{2} \backslash e\right)} c(\bar{e})$ because $\prod_{\bar{e} \in S_{1}} c(\bar{e})=\prod_{\bar{e} \in S_{2}} c(\bar{e})$ and $c(e) \neq 0$.

* For each $e \in S_{1} \cap S_{2}$ and for each $\alpha>0$ :

$$
\begin{aligned}
f_{c_{\alpha, e}}\left(S_{1}\right) & =\prod_{\left.\bar{e} \in S_{1} \backslash e\right\}} c(\bar{e}) \cdot(c(e)+\alpha) \\
& =\prod_{\bar{e} \in S_{2} \backslash e e} c(\bar{e}) \cdot(c(e)+\alpha) \\
& =f_{c_{\alpha, e}}\left(S_{2}\right)
\end{aligned}
$$

Lemma 5: (1) holds for a cost function of type MAX.

Proof: The case $u_{S_{1}}(e)=+\infty$ can also be shown analogously to the proof of Lemma 3.

In the following, we assume that $u_{S_{1}}(e) \neq+\infty$ and $u_{S_{2}}(e) \neq+\infty$.

Because of the definition of $u_{S_{1}}(e)$ and Condition 1, the following statements obviously hold for all $\mathcal{E}>0$ :

* $\quad S_{1}$ is an optimal solution of $P_{u_{S_{1}}(e), e}$.

* $\quad S_{1}$ isn't an optimal solution of $P_{u_{S_{1}}(e)+\varepsilon, e}$ although feasible solution.

It follows that $f_{c_{u_{S_{1}}(e), e}}\left(S_{1}\right)=c(e)+u_{S_{1}}(e)$ must hold.
Otherwise, $f_{c_{u_{1}}(e), e}\left(S_{1}\right)>c(e)+u_{S_{1}}(e)$ would hold and the costs of $e$ could be increased by some constant $\varepsilon>0$ without violating the optimality of $S_{1}$. Furthermore, we have:

$$
\begin{aligned}
f_{c}\left(S_{2}\right) & =f_{c}\left(S_{1}\right) \quad \text { as } S_{1}, S_{2} \in D^{\star} \\
& \leq f_{c_{u_{1}(e) e}}\left(S_{1}\right) \quad \text { monotonicity of the cost function } \\
& =c(e)+u_{S_{1}}(e)
\end{aligned}
$$

Thus, as the cost function we consider in this lemma is of type MAX, the costs of $S_{2}$ with respect to $P_{u_{1}(e), e}$ is determined by element $e$ as $e \in S_{2}$ and the costs of all the other elements of $S_{2}$ are less than or equal $c(e)+u_{S_{1}}(e)$, i.e.,

$f_{c_{u_{S_{1}}(e), e}}\left(S_{2}\right)=c(e)+u_{S_{1}}(e)=f_{c_{u_{S_{1}}(e), e}}\left(S_{1}\right)$

As $S_{1}$ is an optimal solution of $P_{u_{S_{1}}(e), e}, S_{2}$ is also an optimal solution of $P_{u_{S_{1}}(e), e}$. Thus, $u_{S_{2}}(e) \geq u_{S_{1}}(e)$ holds. The relation $u_{S_{2}}(e) \leq u_{S_{1}}(e)$ can be shown analogously.

\section{Proof of Theorem 3}

Lemma 6: Let $S_{1}, S_{2} \subseteq 2^{E}$ be two subsets of $E$, $e \in S_{1} \cap S_{2}$ and $\alpha>0$. It holds:

$f_{c}\left(S_{1}\right) \geq f_{c}\left(S_{2}\right) \Rightarrow f_{c_{\alpha, e}}\left(S_{1}\right) \geq f_{c_{\alpha, e}}\left(S_{2}\right)$

Note that the above implication even holds for all $\alpha \in \mathbf{R}$, if the cost function is either of type $\Sigma$ or $\Pi$.

Proof: We prove the lemma by case differentiation.

* [The cost function is of type $\Sigma$ ]

$$
\begin{aligned}
f_{c_{\alpha, e}}\left(S_{1}\right) & =\alpha+f_{c}\left(S_{1}\right) \\
& \geq \alpha+f_{c}\left(S_{2}\right) \\
& =f_{c_{\alpha, e}}\left(S_{2}\right)
\end{aligned}
$$

Thus, (4) holds even for all $\alpha \in \mathbf{R}$.

* [The cost function is of type $\Pi]$

$$
\begin{aligned}
f_{c_{\alpha, e}}\left(S_{1}\right) & =(c(e)+\alpha) \cdot \frac{f_{c}\left(S_{1}\right)}{c(e)} \quad \text { as } c(e) \neq 0 \\
& \geq(c(e)+\alpha) \cdot \frac{f_{c}\left(S_{2}\right)}{c(e)} \\
& =f_{c_{\alpha, e}}\left(S_{2}\right)
\end{aligned}
$$

Thus, (4) holds even for all $\alpha \in \mathbf{R}$.

* [The cost function is of type MAX ]

There are three sub-cases to distinguish:

Case 1: $f_{c}\left(S_{1}\right) \geq c(e)+\alpha$ and $f_{c}\left(S_{2}\right) \geq c(e)+\alpha$

Because of $\alpha>0$, it follows:

$f_{c_{\alpha, e}}\left(S_{1}\right)=f_{c}\left(S_{1}\right), f_{c_{\alpha, e}}\left(S_{2}\right)=f_{c}\left(S_{2}\right)$

so that (4) obviously holds. 
Case 2: $f_{c}\left(S_{1}\right) \geq c(e)+\alpha$ and $f_{c}\left(S_{2}\right)<c(e)+\alpha$

Because of $\alpha>0$, it follows:

$f_{c_{\alpha, e}}\left(S_{1}\right)=f_{c}\left(S_{1}\right) \geq c(e)+\alpha=f_{c_{\alpha, e}}\left(S_{2}\right)$

Case 3: $f_{c}\left(S_{1}\right)<c(e)+\alpha$

and $f_{c}\left(S_{2}\right)<c(e)+\alpha$

It follows:

$$
f_{c_{\alpha, e}}\left(S_{1}\right)=c(e)+\alpha=f_{c_{\alpha, e}}\left(S_{2}\right)
$$

Now, we prove Theorem 3.

Let $e \in E$ be with $u_{P}(e) \notin\{$ UNDEFINED, $+\infty\}$, $\varepsilon>0$ and $S \in D$ with $e \in S$ a feasible solution of $P_{u_{P}(e)+\varepsilon, e}$. We show that $S$ isn't an optimal solution of $P_{u_{p}(e)+\varepsilon, e} \cdot$

Because of Condition $1, S$ is a feasible solution of $P$. Now, we can distinguish two cases:

* [S is optimal with respect to $P]$ : Then, $u_{S}(e)$ is defined and because of the definition of upper tolerance and Theorem 2, $S$ isn't an optimal solution of $P_{u_{P}(e)+\varepsilon, e}$

* [S isn't optimal with respect to $P$ ] : Because of $u_{P}(e) \neq$ UNDEFINED, there is an optimal solution $S^{\star}$ of $P$ with $e \in S^{\star}$. As just proven, $S^{\star}$ is not optimal with respect to $P_{u_{P}(e)+\varepsilon, e}$. As $S \notin D^{\star}$, it follows: $f_{c}(S)>f_{c}\left(S^{\star}\right)$. As $e \in S \cap S^{\star}$, Lemma 6 can be applied: $f_{c_{u p(e)+e, e}}(S) \geq f_{c_{c_{u p}(e)+e, e}}\left(S^{\star}\right)$. Hence, $S$ cannot be optimal with respect to $P_{u_{p}(e)+\varepsilon, e}$ as its costs are greater than or equal those of $S^{\star}$ which isn't an optimal solution of $P_{u_{p}(e)+\varepsilon, e}$.

Proof of Theorem 4: Theorem 4 follows from the following three lemma, Lemma 7, 8 and 9.

Lemma 7. Let the cost function be of type $\sum$. For each single element $e \in E$ which is contained in at least one optimal solution $S^{\star}$ of $P$, the upper tolerance of $e$ is given by:

$u_{P}(e)=f_{c}\left(D_{-}^{\star}(e)\right)-f_{c}(P)$

Proof: First, let us prove that $u_{P}(e) \geq f_{c}\left(D_{-}^{\star}(e)\right)-f_{c}(P)$ holds.

If $u_{P}(e)=+\infty$, the above relation is obvious. Thus, we can assume $u_{p}(e) \neq+\infty$ in the following. The equation $f_{c_{u_{P}(e)+\varepsilon, e}}\left(D_{-}^{\star}(e)\right)=f_{c}\left(D_{-}^{\star}(e)\right)$ holds for each $\varepsilon>0$, as only the costs of element $e$ are increased.
By Theorem 3, for all $\varepsilon>0$ there is no feasible solution $S \in D$ with $e \in S$ which is an optimal solution of $P_{u_{p}(e)+\varepsilon, e}$, i.e., $f_{c_{u p(e)+\varepsilon, e}}\left(D_{-}^{\star}(e)\right)<f_{c_{u p(e)+\varepsilon, e}}\left(S^{\star}\right)$ holds, as $e \in S^{\star}$. Hence, for all $\varepsilon>0$

$$
\begin{aligned}
f_{c}\left(D_{-}^{\star}(e)\right) & =f_{c_{u_{P P}(e)+\varepsilon, e}}\left(D_{-}^{\star}(e)\right) \\
& <f_{c_{u p(e)+e, e}}\left(S^{\star}\right) \\
& =f_{c}(P)+u_{P}(e)+\mathcal{E}
\end{aligned}
$$

Thus, $f_{c}\left(D_{-}^{\star}(e)\right) \leq f_{c}(P)+u_{P}(e)$ holds which is equivalent to $f_{c}\left(D_{-}^{\star}(e)\right)-f_{c}(P) \leq u_{P}(e)$.

Now, let us prove the other direction, namely $u_{P}(e) \leq f_{c}\left(D_{-}^{\star}(e)\right)-f_{c}(P)$.

Let $\beta(e):=f_{c}\left(D_{-}^{\star}(e)\right)-f_{c}(P)$.

We can assume that $\beta(e) \neq+\infty$, as otherwise the assertion is proven obviously. Increasing the costs of $e$ by $\beta(e)+\varepsilon$ with $\varepsilon>0$ lets increase the costs of the formerly optimal solution $S^{\star}$ to

$$
\begin{aligned}
f_{c_{\beta(e)+\varepsilon, e}}\left(S^{\star}\right) & =f_{c}\left(S^{\star}\right)+\beta(e)+\varepsilon \\
& =f_{c}(P)+\left(f_{c}\left(D_{-}^{\star}(e)\right)-f_{c}(P)\right)+\varepsilon \\
& =f_{c}\left(D_{-}^{\star}(e)\right)+\varepsilon \\
& >f_{c}\left(D_{-}^{\star}(e)\right) \\
& =f_{c_{\beta(e)+e, e}}\left(D_{-}^{\star}(e)\right)
\end{aligned}
$$

Thus, $S^{\star}$ is no optimal solution of $P_{\beta(e)+\varepsilon, e}$ and $u_{P}(e)<\beta(e)+\varepsilon$. It follows:

$$
u_{P}(e) \leq \beta(e)=f_{c}\left(D_{-}^{\star}(e)\right)-f_{c}(P)
$$

Lemma 8: Let the cost function be of type $\Pi$. For each single element $e \in E$ which is contained in at least one optimal solution $S^{\star}$ of $P$, the upper tolerance of $e$ is given by:

$u_{P}(e)=\frac{f_{c}\left(D_{-}^{\star}(e)\right)-f_{c}(P)}{f_{c}(P)} \cdot c(e)$

Proof: First, let us prove that $u_{P}(e) \geq \frac{f_{c}\left(D_{-}^{\star}(e)\right)-f_{c}(P)}{f_{c}(P)} \cdot c(e)$ holds.

We only have to prove the relation for $u_{P}(e) \neq+\infty$.

The equation $f_{c_{u p(e)+\varepsilon, e}}\left(D_{-}^{\star}(e)\right)=f_{c}\left(D_{-}^{\star}(e)\right)$ holds for each $\varepsilon>0$. By Theorem 3, for all $\varepsilon>0$ there is no feasible solution $S \in D$ with $e \in S$ which is an optimal solution of $P_{u_{p}(e)+\varepsilon, e}$, i.e., $f_{c_{u_{p}(e)+\varepsilon, e}}\left(D_{-}^{\star}(e)\right)<f_{c_{u p(e)+\varepsilon, e}}\left(S^{\star}\right)$

holds, as $e \in S^{\star}$. Hence, for all $\varepsilon>0$

$$
\begin{aligned}
f_{c}\left(D_{-}^{\star}(e)\right) & =f_{c_{u p(e)+\varepsilon, e}}\left(D_{-}^{\star}(e)\right) \\
& <f_{c_{u_{P}(e)+\varepsilon, e}}\left(S^{\star}\right)
\end{aligned}
$$




$$
\begin{aligned}
& =\prod_{\bar{e} \in S^{\star} \backslash\{e\}} c(\bar{e}) \cdot\left(c(e)+u_{P}(e)+\mathcal{E}\right) \\
& =\prod_{\bar{e} \in S^{\star}} c(\bar{e})+\left(u_{P}(e)+\varepsilon\right) \cdot \prod_{\bar{e} \in S^{\star} \backslash\{e\}} c(\bar{e}) \\
& =\prod_{\bar{e} \in S^{\star}} c(\bar{e})+\left(u_{P}(e)+\varepsilon\right) \cdot \frac{1}{c(e)} \cdot \prod_{\bar{e} \in S^{\star}} c(\bar{e}) \\
& =f_{c}\left(S^{\star}\right)+\left(u_{P}(e)+\varepsilon\right) \cdot \frac{1}{c(e)} \cdot f_{c}\left(S^{\star}\right) \\
& =f_{c}(P)+\left(u_{P}(e)+\varepsilon\right) \cdot \frac{1}{c(e)} \cdot f_{c}(P)
\end{aligned}
$$

Thus, $\quad \frac{f_{c}\left(D_{-}^{\star}(e)\right)-f_{c}(P)}{f_{c}(P)} \cdot c(e)<u_{P}(e)+\mathcal{E} \quad$ holds which implies:

$$
\frac{f_{c}\left(D_{-}^{\star}(e)\right)-f_{c}(P)}{f_{c}(P)} \cdot c(e) \leq u_{P}(e)
$$

Now, let us prove the other direction, namely $u_{P}(e) \leq \frac{f_{c}\left(D_{-}^{\star}(e)\right)-f_{c}(P)}{f_{c}(P)} \cdot c(e)$. Let

$$
\beta(e):=\frac{f_{c}\left(D_{-}^{\star}(e)\right)-f_{c}(P)}{f_{c}(P)} \cdot c(e)
$$

Once again, we can assume that $\beta(e) \neq+\infty$. Increasing the costs of $e$ by $\beta(e)+\varepsilon$ with $\varepsilon>0$ lets increase the costs of the formerly optimal solution $S^{\star}$ to

$$
\begin{aligned}
f_{c_{\beta(e)+\varepsilon, e}}\left(S^{\star}\right) & =\frac{f_{c}\left(S^{\star}\right)}{c(e)} \cdot(c(e)+\beta(e)+\varepsilon) \\
& >\frac{f_{c}\left(S^{\star}\right)}{c(e)} \cdot(c(e)+\beta(e)) \\
& =f_{c}\left(S^{\star}\right)+\beta(e) \cdot \frac{f_{c}\left(S^{\star}\right)}{c(e)} \\
& =f_{c}(P)+\frac{f_{c}\left(D_{-}^{\star}(e)\right)-f_{c}(P)}{f_{c}(P)} \cdot c(e) \cdot \frac{f_{c}(P)}{c(e)} \\
& =f_{c}\left(D_{-}^{\star}(e)\right) \\
& =f_{c_{\beta(e)+\varepsilon, e}}\left(D_{-}^{\star}(e)\right)
\end{aligned}
$$

Thus, $S^{\star}$ is no optimal solution of $P_{\beta(e)+\varepsilon, e}$ and $u_{P}(e)<\beta(e)+\varepsilon$. It follows:

$$
u_{P}(e) \leq \beta(e)=\frac{f_{c}\left(D_{-}^{\star}(e)\right)-f_{c}(P)}{f_{c}(P)} \cdot c(e)
$$

Lemma 9: Let the cost function be of type MAX . For each single element $e \in E$ which is contained in at least one optimal solution $S^{\star}$ of $P$, the upper tolerance of $e$ is given by:

$$
u_{P}(e)=f_{c}\left(D_{-}^{\star}(e)\right)-c(e)
$$

Proof: First, let us prove that $u_{P}(e) \geq f_{c}\left(D_{-}^{\star}(e)\right)-c(e)$ holds.

We only have to prove the relation for $u_{P}(e) \neq+\infty$. The equation $f_{c_{u_{P}(e)+\varepsilon, e}}\left(D_{-}^{\star}(e)\right)=f_{c}\left(D_{-}^{\star}(e)\right)$ holds for each $\varepsilon>0$.

Furthermore $f_{c_{u_{P}(e)+\varepsilon, e}}\left(S^{\star}\right)=c(e)+u_{P}(e)+\varepsilon$ holds, as $S^{\star}$ is no optimal solution of $P_{u_{P}(e)+\varepsilon, e}$.

By Theorem 3, for all $\varepsilon>0$ there is no feasible solution $S \in D$ with $e \in S$ which is an optimal solution of $P_{u_{P}(e)+\varepsilon, e}$, i.e., $f_{c_{u_{P}(e)+\varepsilon, e}}\left(D_{-}^{\star}(e)\right)<f_{c_{u_{P}(e)+\varepsilon, e}}\left(S^{\star}\right)$ holds, as $e \in S^{\star}$. Hence, for all $\varepsilon>0$

$$
\begin{aligned}
f_{c}\left(D_{-}^{\star}(e)\right) & =f_{c_{u_{P}(e)+\varepsilon, e}}\left(D_{-}^{\star}(e)\right) \\
& <f_{c_{u_{P}(e)+\varepsilon, e}}\left(S^{\star}\right) \\
& =c(e)+u_{P}(e)+\varepsilon
\end{aligned}
$$

Thus, $\quad f_{c}\left(D_{-}^{\star}(e)\right)-c(e)<u_{P}(e)+\varepsilon$ holds which implies: $f_{c}\left(D_{-}^{\star}(e)\right)-c(e) \leq u_{P}(e)$.

Now, let us prove the other direction, namely $u_{P}(e) \leq f_{c}\left(D_{-}^{\star}(e)\right)-c(e)$.

Let $\beta(e):=f_{c}\left(D_{-}^{\star}(e)\right)-c(e)$.

We can assume that $\beta(e) \neq+\infty$. For the optimal solution $S^{\star} \in D^{\star}$ with $e \in S^{\star}$, the following holds for all $\varepsilon>0$ :

$$
\begin{aligned}
& f_{c_{\beta(e)+\varepsilon, e}}\left(S^{\star}\right) \\
= & \max \{\max \{c(\bar{e}) ; \\
\left.\left.\bar{e} \in S^{\star} \backslash\{e\}\right\}, c(e)+\beta(e)+\varepsilon\right\} & \max \left\{\max \left\{c(\bar{e}) ; \bar{e} \in S^{\star} \backslash\{e\}\right\},\right. \\
& \left.\quad c(e)+f_{c}\left(D_{-}^{\star}(e)\right)-c(e)+\varepsilon\right\} \\
= & \max \left\{\max \left\{c(\bar{e}) ; \bar{e} \in S^{\star} \backslash\{e\}\right\}, f_{c}\left(D_{-}^{\star}(e)\right)+\varepsilon\right\} \\
> & f_{c}\left(D_{-}^{\star}(e)\right)
\end{aligned}
$$

Thus, $S^{\star}$ is no optimal solution of $P_{\beta(e)+\varepsilon, e}$ and $u_{P}(e)<\beta(e)+\varepsilon$ for all $\varepsilon>0$. It follows:

$$
u_{P}(e) \leq \beta(e)=f_{c}\left(D_{-}^{\star}(e)\right)-c(e)
$$

Proof of Theorem 5: Let $e$ be a single element of $E$. First, let $e$ be in each feasible solution of $P$. Then

$$
f_{c}\left(D_{-}^{\star}(e)\right)=f_{c}(\varnothing)=+\infty=f_{c_{+\infty, e}}(P)
$$

So assume that there is at least one feasible solution $S$ with $e \notin S$. Let $S_{+\infty}^{*}$ be an optimal solution of $P_{+\infty, e}$. Because of the assumption and Condition 1 , $e \notin S_{+\infty}^{*}$. So

$$
f_{c}\left(D_{-}^{\star}(e)\right)=f_{c_{+\infty, e}}\left(D_{-}^{\star}(e)\right)=f_{c_{+\infty, e}}(P)
$$


Proof of Theorem 6: By Theorem 4,

$u_{P}(e)=\left\{\begin{array}{cl}f_{c}\left(D_{-}^{\star}(e)\right)-f_{c}(P) & \text {,if } f_{c} \text { is of type } \sum \\ \frac{f_{c}\left(D_{-}^{\star}(e)\right)-f_{c}(P)}{f_{c}(P)} \cdot c(e) & \text {,if } f_{c} \text { is of type } \Pi\end{array}\right.$ holds.

First, we prove the direction " $\Rightarrow$ ". Because $e$ is contained in every optimal solution, the costs of a feasible solution not containing $e$ is greater than the costs of an optimal solution, i.e., $f_{c}\left(D_{-}^{\star}(e)\right)>f_{c}(P)$. Hence, $u_{P}(e)>0$.

Now, let us prove the other direction. Let $u_{P}(e)>0$. Assume that there is an optimal solution $S^{\star}$ with $e \notin S^{\star}$. By this, $f_{c}\left(D_{-}^{\star}(e)\right)=f_{c}(P)$ and $u_{P}(e)=0$ follows, which is a contradiction to $u_{P}(e)>0$.

Proof of Remark 1: Let the cost function be of type MAX.

For the direction " $\Rightarrow$ " let $e$ be contained in every optimal solution. Thus

$f_{c}\left(D_{-}^{\star}(e)\right)>f_{c}(P) \geq c(e)$

Hence, $u_{P}(e)>0$ with Theorem 4.

For the other direction consider the following combinatorial minimization problem $P=\left(E, D, c, f_{c}\right)$ defined by:

* $E=\{v, x, y, z\}$ with $c(v)=1, c(x)=c(y)=2$ and $c(z)=3$

* $D=\{\{p, q\} ; p, q \in E$ and $p \neq q\}$

* $f_{c}$ is a cost function of type MAX

Obviously, there are three optimal solutions, namely $\{v, x\},\{v, y\}$ and $\{x, y\}$. The costs $f_{c}\left(D_{-}^{\star}(v)\right)$ of the best feasible solution which doesn't contain $v$ is 2 . By Theorem 4, the upper tolerance of $v$ with respect to $P$ is given by $f_{c}\left(D_{-}^{\star}(v)\right)-c(v)$. Hence, $u_{P}(v)=1>0$ although $\{x, y\}$ is an optimal solution of $P$ which doesn't contain $v$.

Proof of Corollary 2: The condition that $u_{P}(e)>0$ for all $e$ with $u_{P}(e) \neq \mathrm{UNDEFINED}$ is equivalent to the condition that $u_{P}(e)>0$ for all $e \in \bigcup_{S^{\star} \in D^{\star}} S^{\star}$. With Theorem 6 this is equivalent to $\bigcup_{S^{\star} \in D^{\star}} S^{\star} \subseteq \bigcap_{S^{\star} \in D^{\star}} S^{\star}$ and equivalent to $\left|D^{\star}\right|=1$.

Proof of Remark 2: Just look at the following combinatorial minimization problem $P=\left(E, D, c, f_{c}\right)$ which doesn't fulfill Condition 1:

$$
\begin{array}{ll}
* & E=\{v, x, y, z\} \quad \text { with } \quad c(v)=c(x)=1 \quad \text { and } \\
& c(y)=c(z)=2 \\
* & D=\{\{p, q\} ; p, q \in E \text { with } p \neq q \text { and } c(p)=c(q)\} \\
* & f_{c} \text { is a cost function of type } \Sigma
\end{array}
$$

Then there is exactly one optimal solution of $P$, namely $S^{\star}=\{v, x\}$. Thus, $f_{c}(P)=f_{c}\left(S^{\star}\right)=2$ holds. Furthermore, there is exactly one feasible solution which doesn't contain element $v$, namely $S=\{y, z\}$. Because of $f_{c}\left(D_{-}^{\star}(v)\right)=f_{c}(S)=4$, the equation $f_{c}\left(D_{-}^{\star}(v)\right)-f_{c}(P)=2$ holds. However, $u_{S^{\star}}(v)=0$, as increasing the costs of $v$ by $\alpha>0$ makes $S^{\star}$ infeasible.

This proves that Theorem 4 doesn't hold if the combinatorial minimization problem $P$ doesn't fulfill Condition 1.

\section{Proofs of the properties of lower tolerances}

Proof of Theorem 7: If there is no feasible solution which contains element $e \in E$, then the costs of $e$ can be decreased by $\alpha>0$ without affecting the costs of a feasible solution. Thus, optimal solutions of $P$ are optimal solutions of $P_{-\alpha, e}$.

To prove the other direction, assume that there is a feasible solution $S \in D$ with $e \in S$. Decreasing the costs of $e$ by some $0<\gamma<\delta_{\max }(e)$ (choose $\gamma$ such that $c(e)-\gamma$ is small enough) results in (note that we consider only a cost function of type $\sum$ and $\Pi$ in this lemma) $f_{c_{-\gamma, e}}(S)<f_{c_{-\gamma, e}}\left(S^{\star}\right)$ and $S^{\star}$ is no optimal solution of $P_{-\gamma, e}$. Thus, the lower tolerance of $e$ with respect to $S^{\star}$ is less than $\gamma$ and $l_{S^{\star}}(e)<\delta_{\max }(e)$.

Proof of Remark 3: The first part of the proof of Theorem 7 shows that the direction " $\Rightarrow$ " holds, even if the cost function is of type MAX.

To prove that the direction " $\Leftarrow$ " doesn't hold for a cost function of type MAX, consider the combinatorial minimization problem $P=\left(E, D, c, f_{c}\right)$ defined by:

* $E=\{v, x, y\}$ with $c(v)=1$ and $c(x)=c(y)=2$

* $D=\{\{p, q\} ; p, q \in E$ and $p \neq q\}$

* $f_{c}$ is a cost function of type MAX

Obviously, each feasible solution is optimal as the costs of each feasible solution is 2 . Decreasing the costs of element $v$ by $\alpha>0$ doesn't affect the costs of 
a feasible solution. Thus, each feasible solution of $P$ is an optimal solution of $P_{-\alpha, v}$. Hence, $l_{\{x, y\}}(v)=+\infty$ although $v$ is contained in the optimal solution $\{v, x\}$.

Proof of Theorem 8: First, consider the case $l_{S_{1}}(e)=\delta_{\max }(e)$. Because of Theorem 3, we have to make a case differentiation.

* [The cost function is either of type $\Sigma$ or $\Pi$ ]

By Theorem 7, e isn't contained in a feasible solution, thus, optimal solutions remain optimal if the costs of $e$ are decreased by $0 \leq \alpha \leq \delta_{\max }(e)$. In particular, $S_{2}$ is an optimal solution of $P_{-\alpha, e}$. Hence, $l_{S_{2}}(e)=\delta_{\max }(e)$.

* [The cost function is of type MAX ]

In this case, $\delta_{\text {max }}(e)=+\infty$.

Now, assume that $l_{S_{2}}(e)=\alpha \neq+\infty$. Then for all $\varepsilon>0, S_{2}$ isn't an optimal solution of $P_{-(\alpha+\varepsilon), e}$. Hence, as $S_{2}$ is optimal with respect to $P$, there is a feasible solution $S \in D$ with

* $\quad e \in S$

* $\quad f_{c_{-(\alpha+e), e}}(S)<f_{c_{-(\alpha+e), e}}\left(S_{2}\right)$

It follows:

$$
\begin{aligned}
& f_{c}\left(S_{1}\right)=f_{c_{-(\alpha+e), e}}\left(S_{1}\right) \quad \text { because of } e \notin S_{1} \\
& \leq f_{c_{-(\alpha+\theta), e}}(S) \quad \text { because of } l_{S_{1}}(e)=+\infty \\
& <f_{c_{-(\alpha+\varepsilon), e}}\left(S_{2}\right) \\
& =f_{c}\left(S_{2}\right) \quad \text { because of } e \notin S_{2}
\end{aligned}
$$

which is a contradiction to the fact that both $S_{1}$ and $S_{2}$ are optimal with respect to $P$. Thus, $l_{S_{2}}(e)$ has to be $+\infty$.

This closes the proof that $l_{S_{1}}(e)=\delta_{\max }(e)$ implies $l_{S_{2}}(e)=\delta_{\max }(e)$.

Now, consider the other case, namely $l_{S_{1}}(e)<\delta_{\max }(e)$. If we decrease the costs of element $e$ by $l_{S_{1}}(e)$, the following statements hold:

* By Condition 1, $S_{1}$ and $S_{2}$ are feasible solutions with respect to $P_{-l_{S_{1}}(e), e}$.

* Because of the definition of lower tolerance, $S_{1}$ is an optimal solution of $P_{-l_{S_{1}}(e), e}$.

* As the costs of neither $S_{1}$ nor $S_{2}$ are affected by decreasing the costs of $e$, we have

$$
\begin{aligned}
f_{c_{-S_{1}(e), e}}\left(S_{2}\right) & =f_{c}\left(S_{2}\right) \\
& =f_{c}\left(S_{1}\right) \quad S_{1} \text { and } S_{2} \text { are optimal w.r.t. } P
\end{aligned}
$$

$$
=f_{c_{-I_{S_{1}}(e), e}}\left(S_{1}\right)
$$

It follows that $S_{2}$ is an optimal solution of $P_{-l_{S_{1}}(e), e}$, too. Hence, $l_{S_{2}}(e) \geq l_{S_{1}}(e)$ holds.

Analogously we can prove $l_{S_{2}}(e) \leq l_{S_{1}}(e)$.

Proof of Theorem 9: Let $e \in E$ with $l_{P}(e) \notin\left\{\mathrm{UNDEFINED}, \delta_{\max }(e)\right\} \quad$ and $\varepsilon \quad$ with $0<\varepsilon<\delta_{\max }(e)-l_{P}(e)$. Further let $S \in D$ with $e \notin S$ be a feasible solution of $P_{-\left(l_{p}(e)+\varepsilon\right), e}$. We show, that $S$ is not an optimal solution of $P_{-\left(l_{p}(e)+\varepsilon\right), e}$.

Because of Condition 1, $S$ is a feasible solution of $P$. We have to distinguish two cases:

* $\quad$ [S is optimal with respect to $P$ ]

In this case, the lower tolerance of $e$ with respect to $S$ is defined and $l_{S}(e)=l_{P}(e)$ holds. By the definition of lower tolerance, $S$ isn't an optimal solution of $P_{-\left(l_{p}(e)+\varepsilon\right), e}$.

* [S isn't optimal with respect to $P$ ]

Because of $l_{P}(e) \neq \mathrm{UNDEFINED}$, there is at least one optimal solution $S^{\star}$ of $P$ with $e \notin S^{\star}$. As just proven, $S^{\star}$ isn't an optimal solution of $P_{-\left(l_{p}(e)+\varepsilon\right), e}$. As $S \notin D^{\star}, f_{c}(S)>f_{c}\left(S^{\star}\right)$ holds and because of $e \notin S \cup S^{\star}$, the costs of neither $S$ nor $S^{\star}$ are changed if the costs of $e$ decrease. Thus $f_{c_{-\left(l_{p}(e)+\varepsilon\right), e}}(S)=f_{c}(S)>f_{c}\left(S^{\star}\right)=f_{c_{-\left(l_{p}(e)+\varepsilon\right), e}}\left(S^{\star}\right)$

holds and $S$ is not an optimal solution of $P_{-\left(l_{p}(e)+\varepsilon\right), e}$.

Proof of Theorem 10: First, let $e$ be not contained in a feasible solution of $P$, i.e., $D_{+}(e)=\varnothing$. Then

$f_{c}\left(D_{+}^{\star}(e)\right)=f_{c}(\varnothing)=+\infty$

Furthermore

$$
\lim _{K \rightarrow+\infty}\left(f_{c_{-K, e}}(P)+K\right)=f_{c}(P)+\lim _{K \rightarrow+\infty} K=+\infty
$$

for a cost function of type $\sum$ and

$$
\begin{aligned}
\lim _{K \rightarrow c(e)^{-}}\left(\frac{f_{c_{-K, e}}(P)}{c(e)-K} \cdot c(e)\right) & =f_{c}(P) \cdot c(e) \cdot \lim _{K \rightarrow c(e)^{-}} \frac{1}{c(e)-K} \\
& =+\infty
\end{aligned}
$$

for a cost function of type $\Pi$ and

$$
\begin{aligned}
\max \{g(e), c(e)\} & =\max \{+\infty, c(e)\} \\
& =+\infty
\end{aligned}
$$

for a cost function of type MAX .

Now, let $e$ be contained in at least one feasible solution of $P$. 
For a cost function of type $\sum$ it holds for all $K$ with $K<c(e): \quad f_{c}\left(D_{+}^{\star}(e)\right)=f_{c_{-K, e}}\left(D_{+}^{\star}(e)\right)+K$

The assertion follows, as for sufficiently large $K$, $f_{c_{-K, e}}\left(D_{+}^{\star}(e)\right)=f_{c_{-K, e}}(P)$.

For a cost function of type $\Pi$ it holds for all $K$ with $0<K<c(e): \quad f_{c}\left(D_{+}^{\star}(e)\right)=\frac{f_{c_{-K, e}}\left(D_{+}^{\star}(e)\right)}{c(e)-K} \cdot c(e)$

Analogously, the assertion follows, as for $K$ sufficiently close to $c(e)$, $f_{c_{-K, e}}\left(D_{+}^{\star}(e)\right)=f_{c_{-K, e}}(P)$.

The assertion for a cost function of type MAX follows from the definition of $g$.

Proof of Theorem 11: The statement follows from the following three lemma, Lemma 10, 11 and 12.

Lemma 10: Let the cost function be of type $\sum$. For each single element $e \in E$ with $l_{P}(e) \neq \mathrm{UNDEFINED,}$ the lower tolerance of $e$ is given by:

$l_{P}(e)=f_{c}\left(D_{+}^{\star}(e)\right)-f_{c}(P)$

Proof: For the case $l_{P}(e)=+\infty$ it follows from Theorem 7 and 8 that $e \in E \backslash \bigcup_{S \in D} S$ and thus, $f_{c}\left(D_{+}^{\star}(e)\right)=f_{c}(\varnothing)=+\infty$. Therefore

$$
f_{c}\left(D_{+}^{\star}(e)\right)-f_{c}(P)=+\infty
$$

Now let $l_{P}(e) \neq+\infty$.

First, let us prove that $l_{P}(e) \geq f_{c}\left(D_{+}^{\star}(e)\right)-f_{c}(P)$ holds. Decreasing the costs of $e$ by $l_{P}(e)+\varepsilon$ with $\varepsilon>0$ decreases the costs of the best feasible solutions which contain $e$ by $l_{P}(e)+\varepsilon$, i.e., $f_{c_{-(l p(e)+\varepsilon), e}}\left(D_{+}^{\star}(e)\right)=f_{c}\left(D_{+}^{\star}(e)\right)-l_{P}(e)-\varepsilon$.

By Theorem 9, for all $\varepsilon>0$ an optimal solution of $P_{-\left(l_{p}(e)+\varepsilon\right), e}$ contains $e$, i.e.,

$f_{c_{-\left(l_{p}(e)+\varepsilon\right), e}}\left(D_{+}^{\star}(e)\right)<f_{c_{-\left(l_{p}(e)+\varepsilon\right), e}}\left(D_{-}^{\star}(e)\right)$

Now, let $S^{\star}$ be an optimal solution of $P$ with $e \notin S^{\star}$. Such a feasible solution $S^{\star}$ exists as $l_{P}(e) \neq$ UNDEFINED holds. Because of

$$
\begin{array}{rlrl}
f_{c_{-(l p(e)+\varepsilon), e}}\left(D_{-}^{\star}(e)\right) & =f_{c}\left(D_{-}^{\star}(e)\right) \\
& =f_{c}\left(S^{\star}\right) & & \text { because } S^{\star} \in D_{-}^{\star}(e) \\
& =f_{c}(P) & & \text { as } S^{\star} \text { is optimal w.r.t. } P
\end{array}
$$

we can conclude:

$$
\begin{aligned}
f_{c}\left(D_{+}^{\star}(e)\right)-l_{P}(e)-\varepsilon & =f_{c_{-(l p(e)+\varepsilon), e}}\left(D_{+}^{\star}(e)\right) \\
& <f_{c_{-\left(l_{P}(e)+\varepsilon\right), e}}\left(D_{-}^{\star}(e)\right) \\
& =f_{c}(P)
\end{aligned}
$$

Thus, $f_{c}\left(D_{+}^{\star}(e)\right)-l_{P}(e) \leq f_{c}(P)$ holds which is equivalent to $f_{c}\left(D_{+}^{\star}(e)\right)-f_{c}(P) \leq l_{P}(e)$.

Now, let us prove the other direction, namely $l_{P}(e) \leq f_{c}\left(D_{+}^{\star}(e)\right)-f_{c}(P)$. Let

$\beta(e):=f_{c}\left(D_{+}^{\star}(e)\right)-f_{c}(P)$

and let $S^{\star}$ be an optimal solution of $P$ with $e \notin S^{\star}$. $S^{\star}$ exists because of $l_{P}(e) \neq$ UNDEFINED. As we have assumed $l_{P}(e) \neq+\infty, D_{+}^{\star}(e)$ is not empty by Theorem 7 and 8 and thus, $\beta(e) \neq+\infty$ holds.

Decreasing the costs of $e$ by $\beta(e)+\varepsilon$ with $\varepsilon>0$ makes the best solutions of $D_{+}^{\star}(e)$ cheaper than the formerly optimal solution $S^{\star}$ which doesn't contain $e$. Indeed, for all $\varepsilon>0$, the following equations hold:

$$
\begin{aligned}
& f_{c_{-(\beta(e)+\varepsilon), e}}\left(D_{+}^{\star}(e)\right) \\
= & f_{c}\left(D_{+}^{\star}(e)\right)-\beta(e)-\varepsilon \\
= & f_{c}\left(D_{+}^{\star}(e)\right)-\left(f_{c}\left(D_{+}^{\star}(e)\right)-f_{c}(P)\right)-\varepsilon \\
= & f_{c}(P)-\varepsilon \\
< & f_{c}(P) \\
= & f_{c}\left(S^{\star}\right) \\
= & f_{c_{-(\beta(e)+\varepsilon), e}}\left(S^{\star}\right)
\end{aligned}
$$

Thus, for all $\varepsilon>0, S^{\star}$ is no optimal solution of $P_{-(\beta(e)+\varepsilon), e}$ and it follows:

$$
l_{P}(e) \leq \beta(e)=f_{c}\left(D_{+}^{\star}(e)\right)-f_{c}(P)
$$

Lemma 11: Let the cost function be of type $\Pi$. For each single element $e \in E$ with $l_{P}(e) \neq$ UNDEFINED, the lower tolerance of $e$ is given by:

$$
l_{P}(e)=\frac{f_{c}\left(D_{+}^{\star}(e)\right)-f_{c}(P)}{f_{c}\left(D_{+}^{\star}(e)\right)} \cdot c(e)
$$

Proof: For the case $l_{P}(e)=c(e)$ it follows from Theorem 7 and 8 that $e \in E \backslash \bigcap_{S \in D} S$ and thus $f_{c}\left(D_{+}^{\star}(e)\right)=f_{c}(\varnothing)=+\infty$

Therefore

$$
\begin{aligned}
\frac{f_{c}\left(D_{+}^{\star}(e)\right)-f_{c}(P)}{f_{c}\left(D_{+}^{\star}(e)\right)} \cdot c(e) & =c(e)-\frac{f_{c}(P)}{f_{c}\left(D_{+}^{\star}(e)\right)} \cdot c(e) \\
& =c(e)
\end{aligned}
$$

Now let $l_{P}(e) \neq c(e)$.

First, let us prove that $l_{P}(e) \geq \frac{f_{c}\left(D_{+}^{\star}(e)\right)-f_{c}(P)}{f_{c}\left(D_{+}^{*}(e)\right)} \cdot c(e)$ holds. 
Decreasing the costs of $e$ by $l_{P}(e)+\varepsilon$ with $\varepsilon>0$ decreases the costs of the best feasible solutions which contain $e$ by $\left(l_{P}(e)+\varepsilon\right) \cdot \frac{1}{c(e)} \cdot f_{c}\left(D_{+}^{\star}(e)\right)$, i.e., $f_{c_{-\left(I_{p}(e)+\varepsilon\right), e}}\left(D_{+}^{\star}(e)\right)=$

$$
f_{c}\left(D_{+}^{\star}(e)\right)-\left(l_{P}(e)+\varepsilon\right) \cdot \frac{1}{c(e)} \cdot f_{c}\left(D_{+}^{\star}(e)\right) .
$$

By Theorem 9, for all $\varepsilon>0$ an optimal solution of $P_{-\left(l_{p}(e)+\varepsilon\right), e}$ contains $e$, i.e.,

$$
f_{c_{-\left(p_{p}(e)+\varepsilon\right), e}}\left(D_{+}^{\star}(e)\right)<f_{c_{-\left(p_{p}(e)+\varepsilon\right), e}}\left(D_{-}^{\star}(e)\right)
$$

Now, let $S^{\star}$ be an optimal solution of $P$ with $e \notin S^{\star}$. Such a feasible solution $S^{\star}$ exists as $l_{P}(e) \neq$ UNDEFINED holds. Because of

$$
\begin{aligned}
f_{c_{-\left(p_{p}(e)+e\right), e}}\left(D_{-}^{\star}(e)\right) & =f_{c}\left(D_{-}^{\star}(e)\right) \\
& =f_{c}\left(S^{\star}\right) \quad \text { because of } S^{\star} \in D_{-}^{\star}(e) \\
& =f_{c}(P) \quad \text { as } S^{\star} \text { is optimal w.r.t. } P
\end{aligned}
$$

we can conclude for all $\varepsilon>0$ :

$$
\begin{aligned}
& f_{c}\left(D_{+}^{\star}(e)\right)-\left(l_{P}(e)+\varepsilon\right) \cdot \frac{1}{c(e)} \cdot f_{c}\left(D_{+}^{\star}(e)\right) \\
= & f_{c_{-(l p(e)+\varepsilon) e}}\left(D_{+}^{\star}(e)\right) \\
< & f_{c_{-\left(l_{p}(e) \varepsilon\right), e}}\left(D_{-}^{\star}(e)\right) \\
= & f_{c}(P)
\end{aligned}
$$

Thus, $f_{c}\left(D_{+}^{\star}(e)\right)-l_{P}(e) \cdot \frac{1}{c(e)} \cdot f_{c}\left(D_{+}^{\star}(e)\right) \leq f_{c}(P)$ holds which is equivalent to

$$
\frac{f_{c}\left(D_{+}^{\star}(e)\right)-f_{c}(P)}{f_{c}\left(D_{+}^{\star}(e)\right)} \cdot c(e) \leq l_{P}(e)
$$

Now, let us prove the other direction, namely $l_{P}(e) \leq \frac{f_{c}\left(D_{+}^{\star}(e)\right)-f_{c}(P)}{f_{c}\left(D_{+}^{\star}(e)\right)} \cdot c(e)$. Let

$$
\beta(e):=\frac{f_{c}\left(D_{+}^{\star}(e)\right)-f_{c}(P)}{f_{c}\left(D_{+}^{\star}(e)\right)} \cdot c(e)
$$

and let $S^{\star}$ be an optimal solution of $P$ with $e \notin S^{\star}$. $S^{\star}$ exists because of $l_{P}(e) \neq$ UNDEFINED. As $l_{P}(e) \neq c(e)$ holds by assumption, $D_{+}^{\star}(e)$ isn't empty (see Theorem 7 and 8) and $f_{c}\left(D_{+}^{\star}(e)\right) \neq+\infty$ holds. Hence

$$
\begin{aligned}
\beta(e) & =\frac{f_{c}\left(D_{+}^{\star}(e)\right)-f_{c}(P)}{f_{c}\left(D_{+}^{\star}(e)\right)} \cdot c(e) \\
& =\left(1-\frac{f_{c}(P)}{f_{c}\left(D_{+}^{\star}(e)\right)}\right) \cdot c(e)<c(e)
\end{aligned}
$$

Decreasing the costs of $e$ by $\beta(e)+\varepsilon$ with $0<\varepsilon<c(e)-\beta(e)$ makes the best solutions of $D_{+}^{\star}(e)$ cheaper than the formerly optimal solution $S^{\star}$ which doesn't contain $e$. Indeed, for all $\varepsilon>0$, the following equations hold:

$$
f_{c_{-(\beta(e)+\varepsilon), e}}\left(D_{+}^{\star}(e)\right)
$$

$$
\begin{aligned}
& =f_{c}\left(D_{+}^{\star}(e)\right)-(\beta(e)+\varepsilon) \cdot \frac{1}{c(e)} \cdot f_{c}\left(D_{+}^{\star}(e)\right) \\
& <f_{c}\left(D_{+}^{\star}(e)\right)-\beta(e) \cdot \frac{1}{c(e)} \cdot f_{c}\left(D_{+}^{\star}(e)\right) \\
& =f_{c}\left(D_{+}^{\star}(e)\right)-\frac{f_{c}\left(D_{+}^{\star}(e)\right)-f_{c}(P)}{f_{c}\left(D_{+}^{\star}(e)\right)} \cdot \frac{c(e)}{c(e)} \cdot f_{c}\left(D_{+}^{\star}(e)\right) \\
& =f_{c}(P) \\
& =f_{c}\left(S^{\star}\right) \\
& =f_{c_{-(\beta(e)+e), e}}\left(S^{\star}\right)
\end{aligned}
$$

Thus, for all $\varepsilon>0, S^{\star}$ is no optimal solution of $P_{-(\beta(e)+\varepsilon), e}$ and it follows:

$$
l_{P}(e) \leq \beta(e)=\frac{f_{c}\left(D_{+}^{\star}(e)\right)-f_{c}(P)}{f_{c}\left(D_{+}^{\star}(e)\right)} \cdot c(e)
$$

Lemma 12: Let the cost function be of type MAX. For each single element $e \in E$ with $l_{P}(e) \neq \mathrm{UNDEFINED}$, the lower tolerance of $e$ is given by:

$l_{P}(e)= \begin{cases}c(e)-f_{c}(P) & , \text { if } g(e)<f_{c}(P) \\ +\infty & , \text { otherwise }\end{cases}$

Proof: Because of $l_{P}(e) \neq$ UNDEFINED there is at least one optimal solution $S^{*}$ of $P$ with $e \notin S^{*}$. Thus, $e$ is not contained in any optimal solution and

$f_{c}(P)=f_{c}\left(D_{-}^{\star}(e)\right)$

holds.

First, let $g(e)<f_{c}(P)$. Assume $c(e)<f_{c}(P)$. Then we obtain a contradiction because of Theorem 10:

$f_{c}\left(D_{+}^{\star}(e)\right)=\max \{g(e), c(e)\}<f_{c}(P) . \quad$ Thus, $c(e) \geq f_{c}(P)$.

It holds for $\alpha \geq 0$ :

$$
\begin{aligned}
& f_{c_{-\alpha, e}}(P)= \min \left\{f_{c_{-\alpha, e}}\left(D_{+}^{\star}(e)\right), f_{c_{-\alpha, e}}\left(D_{-}^{\star}(e)\right)\right\} \\
&= \min \left\{\max \{g(e), c(e)-\alpha\}, f_{c}(P)\right\} \\
& \text { because of Theorem } 10 \text { and }(5) \\
& \begin{cases}=f_{c}(P), & \text { if } \alpha \leq c(e)-f_{c}(P) \\
<f_{c}(P), & \text { if } \alpha>c(e)-f_{c}(P)\end{cases} \\
& \begin{cases}=f_{c}\left(S^{*}\right), & \text { if } \alpha \leq c(e)-f_{c}(P) \\
<f_{c}\left(S^{*}\right), & \text { if } \alpha>c(e)-f_{c}(P)\end{cases}
\end{aligned}
$$

From

$f_{c_{-\alpha, e}}(P)=f_{c}\left(S^{*}\right)=f_{c_{-\alpha, e}}\left(S^{*}\right)$ for $\quad \alpha \leq c(e)-f_{c}(P)$ it follows: $l_{P}(e) \geq c(e)-f_{c}(P)$.

From $f_{c_{-\alpha, e}}(P)<f_{c}\left(S^{*}\right) \quad$ for $\quad \alpha>c(e)-f_{c}(P) \quad$ it 
follows: $l_{P}(e) \leq c(e)-f_{c}(P)$.

So we have: $l_{P}(e)=c(e)-f_{c}(P)$.

Now, let $g(e) \geq f_{c}(P)$. From (2) and (5) it follows:

$$
\begin{aligned}
f_{c_{-\infty, e}}(P) & =\min \left\{g(e), f_{c}\left(D_{-}^{\star}(e)\right)\right\} \\
& =\min \left\{g(e), f_{c}(P)\right\} \\
& =f_{c}(P) \\
& =f_{c}\left(S^{*}\right) \\
& =f_{c_{-\infty, e}}\left(S^{*}\right)
\end{aligned}
$$

$l_{P}(e)=+\infty$ follows from the definition of lower tolerance.

Proof of Theorem 12: By Theorem 11,

$$
l_{P}(e)=\left\{\begin{array}{cl}
f_{c}\left(D_{+}^{\star}(e)\right)-f_{c}(P) & \text {,if } f_{c} \text { is of type } \Sigma \\
\frac{f_{c}\left(D_{+}^{\star}(e)\right)-f_{c}(P)}{f_{c}\left(D_{+}^{\star}(e)\right)} \cdot c(e) & \text {, if } f_{c} \text { is of type } \Pi
\end{array}\right.
$$

holds, if $1_{P}(e) \neq$ UNDEFINED.

First, we prove the direction " $\Rightarrow$ ". Because $e$ isn't contained in any optimal solution, $l_{P}(e) \neq$ UNDEFINED and the costs of a feasible solution which contains $e$ is greater than the costs of an optimal solution, i.e., $f_{c}\left(D_{+}^{\star}(e)\right)>f_{c}(P)$. Hence, $l_{P}(e)$ is greater than 0 .

Now, let us prove the other direction. Let $l_{P}(e)>0$. Assume that there is an optimal solution $S^{\star}$ with $e \in S^{\star}$. By this, $f_{c}\left(D_{+}^{\star}(e)\right)=f_{c}(P)$ and $l_{P}(e)=0$ follows which is a contradiction.

Proof of Remark 4: Let the cost function be of type MAX .

To prove the direction " $\Rightarrow$ " let $e$ be not contained in any optimal solution. Then $l_{P}(e) \neq$ UNDEFINED. Assume that $l_{P}(e)=0$. By Theorem 11, it follows $c(e)=f_{c}(P)$ and $g(e)<f_{c}(P)$. With the definition of $g$ we have: $f_{c}\left(D_{+}^{\star}(e)\right)=f_{c}(P)$ which is a contradiction to the assumption that $e$ is not contained in any optimal solution.

To prove that the direction " $\Leftarrow$ " doesn't hold, consider the following combinatorial minimization problem $P=\left(E, D, c, f_{c}\right)$ defined by:

* $E=\{v, x, y\}$ with $c(v)=1, c(x)=1, c(y)=1$

* $D=\{\{v, x\},\{v, y\},\{x, y\}\}$

* $f_{c}$ is a cost function of type MAX
Each feasible solution is an optimal solution, i.e, $E=\bigcup_{S^{\star} \in D^{\star}} S^{\star} \quad$ and so $E \backslash \bigcup_{S^{\star} \in D^{\star}} S^{\star}=\varnothing$. Furthermore it holds: $l_{P}(v)=+\infty$.

\section{Proofs of the relationship between upper and lower tolerances}

Proof of Lemma 1: Obviously, $S$ is also the only optimal solution of $P$.

We make the following case differentiation:

* $\quad[\mathbf{S}=\mathbf{E}]$

As every single element of $E$ is contained in each feasible solution, each single element $e \in E$ has the upper tolerance $u_{S}(e)=+\infty$ because of

Theorem 1. As the only optimal solution contains each single element of $E$, the lower tolerance isn't defined for a single element of $E$, i.e., $l_{P}(e)=\mathrm{UNDEFINED}$ for all $e \in E$ and $\left\{l_{P}(e)\right.$; $e \in E \quad$ and $\left.\quad l_{P}(e) \neq \quad \mathrm{UNDEFINED}\right\}=\varnothing \quad$ holds. Hence

$$
\begin{aligned}
& l_{P, \min } \\
= & \min \left\{l_{P}(e) ; e \in E \text { and } l_{P}(e) \neq\right. \\
& \mathrm{UNDEFINED}\} \\
= & \min \varnothing \\
= & +\infty \\
= & \min \{+\infty\} \\
= & \min \left\{u_{P}(e) ; e \in E\right\} \\
= & \min \left\{u_{P}(e) ; e \in E \text { and } u_{P}(e) \neq\right. \\
& \mathrm{UNDEFINED}\} \\
= & u_{P, \min }
\end{aligned}
$$$$
* \quad[\mathbf{S}=\varnothing]
$$

As the only optimal solution is empty, the upper tolerance isn't defined for a single element of $E$, i.e., $u_{P}(e)=\mathrm{UNDEFINED}$ holds for all $e \in E$. This implies

$$
\begin{aligned}
& \left\{u_{P}(e) ; e \in E \text { and } u_{P}(e) \neq \mathrm{UNDEFINED}\right\}=\varnothing \text { and } \\
& \begin{aligned}
u_{P, \text { min }} & =\min \left\{u_{P}(e) ; e \in E \text { and } u_{P}(e) \neq \mathrm{UNDEFINED}\right\} \\
& =\min \varnothing \\
& =+\infty
\end{aligned}
\end{aligned}
$$

As the only feasible solution is empty, Theorem 7 can be applied to each single element $e \in E$. Hence, $l_{P}(e)=\delta_{\max }(e)$ holds for all $e \in E$. This implies: $l_{P, \min }=\Delta_{P, \min }$.

$* \quad[\mathbf{S} \neq \mathbf{E}$ and $\mathbf{S} \neq \varnothing]$

For each single element $e_{\text {out }} \in E \backslash S$, the lower tolerance $l_{P}\left(e_{\text {out }}\right)$ is $\delta_{\max }\left(e_{\text {out }}\right)$ and the upper tolerance of $e_{\text {out }}$ isn't defined. Analogously, for 
every single element $e_{i n} \in S$, the upper tolerance $u_{P}\left(e_{i n}\right)$ is $+\infty$ and the lower tolerance of $e_{i n}$ isn't defined. Thus, $u_{P, \min }=+\infty$ and $l_{P, \min } \geq \Delta_{P, \min }$ hold.

Proof of Lemma 2: In the following, let $S^{\star}$ be the optimal solution of $P$.

First, we prove $l_{P, \min } \leq u_{P, \min }$. Let $S \in D \backslash\left\{S^{\star}\right\}$ be a feasible (but non-optimal) solution. By assumption, $S^{\star} \nsubseteq S$, i.e., there is an element $e^{\star} \in S^{\star} \backslash S$. Because of Theorem 1 and $e^{\star} \notin S \in D, u_{P}\left(e^{\star}\right) \neq+\infty$.

Now, let $e^{\star}$ be an element of $S^{\star}$ with $u_{P}\left(e^{\star}\right) \neq+\infty$. Because of the definition of upper tolerance, for all $\varepsilon>0$, solution $S^{\star}$ is not an optimal solution of $P_{u_{P}\left(e^{\star}\right)+\varepsilon, e^{\star}}$. By Theorem 3 there is a solution $S^{\prime} \in D \backslash\left\{S^{\star}\right\}$ with $e^{\star} \notin S^{\prime}$ and

$$
f_{c_{u_{P}\left(e^{\star}\right)+\varepsilon, e^{\star}}}\left(S^{\prime}\right)<f_{c_{u_{P}\left(e^{\star}\right)+\varepsilon, e^{\star}}}\left(S^{\star}\right)
$$

Again, $S^{\prime} \nsubseteq S^{\star}$ holds, i.e., there is an element $e^{\prime} \in S^{\prime} \backslash S^{\star}$. Now, decreasing the costs of element $e^{\prime}$ by $u_{P}\left(e^{\star}\right)+\varepsilon$ also implies that $S^{\star}$ is not an optimal solution any more. In fact

$$
\begin{aligned}
& f_{c_{-\left(u_{P}\left(e^{\star}\right)+\varepsilon\right), e^{\prime}}}\left(S^{\prime}\right)=f_{c}\left(S^{\prime}\right)-\left(u_{P}\left(e^{\star}\right)+\mathcal{E}\right) \\
& \text { as } e^{\prime} \in S^{\prime} \\
& =f_{c_{u p\left(e^{\star}\right)+\varepsilon, e^{\star}}}\left(S^{\prime}\right)-\left(u_{P}\left(e^{\star}\right)+\varepsilon\right) \\
& \text { as } e^{\star} \notin S^{\prime} \\
& <f_{c_{u_{P}\left(e^{\star}\right)+\varepsilon, e^{\star}}}\left(S^{\star}\right)-\left(u_{P}\left(e^{\star}\right)+\varepsilon\right) \\
& \text { because of (6) } \\
& =\left(f_{c}\left(S^{\star}\right)+u_{P}\left(e^{\star}\right)+\varepsilon\right)-\left(u_{P}\left(e^{\star}\right)+\varepsilon\right) \\
& \text { as } e^{\star} \in S^{\star} \\
& =f_{c}\left(S^{\star}\right) \\
& =f_{c_{-\left(u_{P}\left(e^{\star}\right)+\varepsilon\right), e^{\prime}}}\left(S^{\star}\right) \\
& \text { as } e^{\prime} \in S^{\star}
\end{aligned}
$$

holds. This implies $1_{P}\left(e^{\prime}\right)<u_{P}\left(e^{\star}\right)+\varepsilon$ for all $\varepsilon>0$, hence, $l_{P}\left(e^{\prime}\right) \leq u_{P}\left(e^{\star}\right)$.

As such an element $e^{\prime}$ exists for each element $e^{\star} \in S^{\star}$ with $u_{P}\left(e^{\star}\right) \neq+\infty, l_{P, \min } \leq u_{P, \min }$ holds.

Now, we prove $u_{P, \min } \leq l_{P, \min }$. Let $S \in D \backslash\left\{S^{\star}\right\}$. By the assumption of the lemma, $S \nsubseteq S^{\star}$, i.e., there is an element $e \in S \backslash S^{\star}$. Because of Theorem 7 and $e \in S \in D, l_{P}(e) \neq+\infty$.

Now, let $e^{\prime}$ be an element of $E \backslash S^{\star}$ with $l_{P}\left(e^{\prime}\right) \neq+\infty$. Because of the definition of lower tolerance, for all $\varepsilon>0$, solution $S^{\star}$ is not an optimal solution of $P_{-\left(l_{P}\left(e^{\prime}\right)+\varepsilon\right), e^{\prime}}$. By Theorem 9, there is a solution $S^{\prime} \in D \backslash\left\{S^{\star}\right\}$ with $e^{\prime} \in S^{\prime}$ and

$f_{c_{-\left(l_{P}\left(e^{\prime}\right)+\varepsilon\right), e^{\prime}}}\left(S^{\prime}\right)<f_{c_{-\left(l_{P}\left(e^{\prime}\right)+\varepsilon\right), e^{\prime}}}\left(S^{\star}\right)$

Because of the assumption, $S^{\star} \mp S^{\prime}$ holds, i.e., there is an element $e^{\star} \in S^{\star} \backslash S^{\prime}$. Now, increasing the costs of element $e^{\star}$ by $l_{P}\left(e^{\prime}\right)+\varepsilon$ also implies that $S^{\star}$ is not an optimal solution any more. In fact

$$
\begin{aligned}
f_{c_{l_{P}\left(e^{\prime}\right)+\varepsilon, e^{\star}}}\left(S^{\prime}\right)= & f_{c}\left(S^{\prime}\right) \\
& \operatorname{as} e^{\star} \notin S^{\prime} \\
= & f_{c_{-\left(l_{P}\left(e^{\prime}\right)+\varepsilon\right), e^{\prime}}}\left(S^{\prime}\right)+\left(l_{P}\left(e^{\prime}\right)+\varepsilon\right) \\
& \operatorname{as} e^{\prime} \in S^{\prime} \\
< & f_{c_{-\left(l_{P}\left(e^{\prime}\right)+\varepsilon\right), e^{\prime}}}\left(S^{\star}\right)+\left(l_{P}\left(e^{\prime}\right)+\varepsilon\right) \\
& \text { because of }(7) \\
= & f_{c}\left(S^{\star}\right)+\left(l_{P}\left(e^{\prime}\right)+\varepsilon\right) \\
& \text { as } e^{\prime} \notin S^{\star} \\
= & f_{c_{l_{P}\left(e^{\prime}\right)+\varepsilon e^{\star}}}\left(S^{\star}\right)-\left(l_{P}\left(e^{\prime}\right)+\varepsilon\right)+\left(l_{P}\left(e^{\prime}\right)+\varepsilon\right) \\
& \operatorname{as} e^{\star} \in S^{\star} \\
= & f_{c_{l_{P}\left(e^{\prime}\right)+\varepsilon, e^{\star}}}\left(S^{\star}\right)
\end{aligned}
$$

holds. This implies $\mathrm{u}_{P}\left(e^{\star}\right)<l_{P}\left(e^{\prime}\right)+\varepsilon$ for all $\varepsilon>0$, hence, $u_{P}\left(e^{\star}\right) \leq l_{P}\left(e^{\prime}\right)$.

As such an element $e^{\star}$ exists for each element $e^{\prime} \in E \backslash S^{\star}$ with $l_{P}\left(e^{\prime}\right) \neq+\infty, u_{P, \min } \leq l_{P, \min }$ holds.

This closes this proof. Note that we have also shown $u_{P, \min } \neq+\infty$.

Proof of Remark 6: Consider the following combinatorial minimization problem $P=\left(E, D, c, f_{c}\right)$ defined by:

$$
\begin{array}{ll}
* & E=\{x, y\} \text { with } c(x)=1, c(y)=1 \\
* & D=\{\{x\},\{x, y\}\} \\
* & f_{c} \text { is a cost function of type } \sum
\end{array}
$$

We have the optimal solution $\{x\}$. It holds:

$u_{P, \min }=u_{P}(x)=+\infty, l_{P}(x)=+\infty, l_{P, \min }=l_{P}(y)=1$.

Proof of Remark 7: Consider the following combinatorial minimization problem $P=\left(E, D, c, f_{c}\right)$

defined by:

* $E=\{v, x, y, z\}$ with $c(v)=1, c(x)=2, c(y)=1$ and $c(z)=1.5$

* $D=\{\{v, x\},\{y, z\}\}$

* $f_{c}$ is a cost function of type $\Pi$ 
By definition, there are two feasible solutions and one optimal solution, namely $\{y, z\}$ whose costs $f_{c}(\{y, z\})$ are 1.5. It holds: $u_{P}(v)=$ UNDEFINED, $u_{P}(x)=$ UNDEFINED,$\quad u_{P}(y)=1 / 3 \quad$ and $u_{P}(z)=0.5$ which implies $u_{P, \min }=1 / 3$ and $l_{P}(v)=0.25$, $l_{P}(x)=0.5, \quad l_{P}(y)=$ UNDEFINED $\quad$ and $l_{P}(z)=\mathrm{UNDEFINED}$ which implies $l_{P, \min }=0.25$. Therefore $u_{P, \text { min }} \neq l_{P, \text { min }}$.

Proof of Remark 8: Consider the following combinatorial minimization problem $P=\left(E, D, c, f_{c}\right)$ defined by:

* $E=\{v, x, y, z\}$ with $c(v)=1, c(x)=2, c(y)=2$ and $c(z)=2$

* $D=\{\{v, x, y\},\{v, x, z\},\{v, y, z\},\{x, y, z\}\}$

* $f_{c}$ is a cost function of type MAX

Each feasible solution is an optimal solution. It holds: $u_{P}(v)=1, u_{P}(x)=0, u_{P}(y)=0$ and $u_{P}(z)=0$ which implies $u_{P, \min }=0$ and $l_{P}(v)=+\infty, l_{P}(x)=+\infty$, $l_{P}(y)=+\infty$ and $l_{P}(z)=+\infty$ which implies $l_{P, \text { min }}=+\infty$. Therefore $u_{P, \text { min }} \neq l_{P, \text { min }}$.

Proof of Theorem 14: First, we show $u_{P, \max } \geq l_{P, \max }$. Because of Theorem 4 there is an $e_{1} \in \bigcup_{S^{\star} \in D^{\star}} S^{\star}$ with $u_{P, \max }+f_{c}(P)=f_{c}\left(D_{-}^{\star}\left(e_{1}\right)\right)$.

Condition a') of the definition of connected implies that there exists $e_{2} \in \bigcup_{S^{\star} \in D^{\star}} S^{\star}, \quad S_{-}^{\star}\left(e_{2}\right) \in D_{-}^{\star}\left(e_{2}\right) \quad$ and $e_{3} \in H$ with $e_{3} \in S_{-}^{\star}\left(e_{2}\right)$ or $S_{-}^{\star}\left(e_{2}\right) \in D_{+}\left(e_{3}\right)$.

Thus

$$
\begin{aligned}
u_{P, \max }+f_{c}(P) & =f_{c}\left(D_{-}^{\star}\left(e_{1}\right)\right) \\
& \geq f_{c}\left(D_{-}^{\star}\left(e_{2}\right)\right) \quad \text { because of Theorem } 4 \\
& =f_{c}\left(S_{-}^{\star}\left(e_{2}\right)\right) \\
& \geq f_{c}\left(D_{+}^{\star}\left(e_{3}\right)\right) \\
& =l_{P}\left(e_{3}\right)+f_{c}(P) \text { because of Theorem 11 } \\
& =l_{P, \max }+f_{c}(P) \quad \text { because of } e_{3} \in H
\end{aligned}
$$

Now, we show $l_{P, \max } \geq u_{P, \max }$. Because of Theorem 11 there is an $e_{1} \in \quad E \backslash \bigcap_{S^{\star} \in D^{\star}} S^{\star} \quad$ with $l_{P, \max }+f_{c}(P)=f_{c}\left(D_{+}^{\star}\left(e_{1}\right)\right)$.

Condition b') of the definition of connected implies that there exists $e_{2} \in E \backslash \bigcap_{S^{\star} \in D^{\star}} S^{\star}, S_{+}^{\star}\left(e_{2}\right) \in D_{+}^{\star}\left(e_{2}\right)$ and $e_{3} \in G$ with $e_{3} \notin S_{+}^{\star}\left(e_{2}\right)$ or $S_{+}^{\star}\left(e_{2}\right) \in D_{-}\left(e_{3}\right)$. Thus

$$
\begin{aligned}
& l_{P, \max }+f_{c}(P)=f_{c}\left(D_{+}^{\star}\left(e_{1}\right)\right) \\
& \geq f_{c}\left(D_{+}^{\star}\left(e_{2}\right)\right) \quad \text { because of Theorem } 11 \\
& =f_{c}\left(S_{+}^{\star}\left(e_{2}\right)\right) \\
& \geq f_{c}\left(D_{-}^{\star}\left(e_{3}\right)\right) \\
& =u_{P}\left(e_{3}\right)+f_{c}(P) \quad \text { because of Theorem } 4 \\
& =u_{P, \max }+f_{c}(P) \quad \text { because of } e_{3} \in G
\end{aligned}
$$

Proof of Remark 9: Consider the following combinatorial minimization problem $P=\left(E, D, c, f_{c}\right)$ defined by:

* $\quad E=\{v, x, y, z\}$ with $c(v)=1, c(x)=2, c(y)=4$ and $c(z)=5$

* $D=\{\{v, x\},\{v, y\},\{v, z\},\{x, y\},\{x, z\},\{y, z\}\}$

* $f_{c}$ is a cost function of type $\Sigma$

The only optimal solution is $\{v, x\}$. It holds:

$$
u_{P}(v)=3, u_{P}(x)=2
$$

which implies $u_{P, \max }=3$ and

$$
l_{P}(y)=2, l_{P}(z)=3
$$

which implies $l_{P, \max }=3$. Therefore

$$
u_{P, \max }=l_{P, \max }
$$

Furthermore it holds:

$$
\begin{gathered}
G=\{v\}, H=\{z\} \\
D_{-}^{\star}(v)=\{\{x, y\}\}, D_{-}^{\star}(x)=\{\{v, y\}\}, \\
D_{+}^{\star}(y)=\{\{v, y\}\}, D_{+}^{\star}(z)=\{\{v, z\}\}
\end{gathered}
$$

As neither condition a') nor condition b') holds, $D$ is not connected.

Proof of Remark 10: Consider the example for the illustration of Theorem 14 for a cost function of type $\Pi$, i.e., the following combinatorial minimization problem $P=\left(E, D, c, f_{c}\right)$ defined by:

$E=\{v, x, y, z\}$ with $c(v)=1, c(x)=2, c(y)=4$ and $c(z)=8$

* $D=\{\{v, x\},\{y, z\}\}$

* $f_{c}$ is a cost function of type $\Pi$

The only optimal solution is $\{v, x\}$.

It holds:

$$
u_{P}(v)=15, u_{P}(x)=30
$$

which implies $u_{P, \max }=30$ and

$$
l_{P}(y)=3.75, l_{P}(z)=7.5
$$

which implies $l_{P, \max }=7.5$ Therefore

Furthermore it holds:

$$
u_{P, \max } \neq l_{P, \max }
$$

$$
G=\{x\}, H=\{z\}
$$

$$
D_{-}^{\star}(v)=\{\{y, z\}\}, D_{-}^{\star}(x)=\{\{y, z\}\},
$$




$$
D_{+}^{\star}(y)=\{\{y, z\}\}, D_{+}^{\star}(z)=\{\{y, z\}\}
$$

As condition a') and condition b') hold, $D$ is connected.

Proof of Remark 11: Consider the example for the illustration of Theorem 14 for a cost function of type MAX, i.e., the following combinatorial minimization problem $P=\left(E, D, c, f_{c}\right)$ defined by:

* $E=\{v, x, y, z\}$ with $c(v)=1, c(x)=2, c(y)=4$ and $c(z)=8$

* $D=\{\{v, x\},\{y, z\}\}$

* $f_{c}$ is a cost function of type MAX

The only optimal solution is $\{v, x\}$. It holds:

$$
\begin{aligned}
& u_{P}(v)=7, u_{P}(x)=6 \\
& \text { which implies } u_{P, \max }=7 \text { and } \\
& l_{P}(y)=+\infty, l_{P}(z)=+\infty \\
& \text { which implies } l_{P, \max }=+\infty \text { Therefore } \\
& u_{P, \max } \neq l_{P, \max } \\
& G=\{v\}, H=\{y, z\} \\
& D_{-}^{\star}(v)=\{\{y, z\}\}, D_{-}^{\star}(x)=\{\{y, z\}\}, \\
& D_{+}^{\star}(y)=\{\{y, z\}\}, D_{+}^{\star}(z)=\{\{y, z\}\}
\end{aligned}
$$

As condition a') and condition b') hold, $D$ is connected.

\section{SUMMARY AND FUTURE RESEARCH DIRECTIONS}

In this paper we have rigorously defined and studied the properties of upper and lower tolerances for a general class of combinatorial optimization problems with three types of objective functions, namely with types $\Sigma, \Pi$, and MAX. Theorems 2 and 8 indicate that the upper and lower tolerances do not depend on a particular optimal solution under the condition that the set of the feasible solutions is independent on the costs of ground elements.

For problems with the objective functions of types $\sum$ and $\Pi$ Theorem 6 implies that the upper tolerances can be considered as an invariant characterizing the structure of the set of all optimal solutions as follows. If all upper tolerances are positive (see Corollary 2), then the set of optimal solutions contains a unique optimal solution. If some upper tolerances are positive and others are zeros, then the set of optimal solutions contains at least two optimal solutions such that the cardinality of their intersection is equal to the number of positive upper tolerances. If all upper tolerances are zeros, then the set of optimal solutions contains at least two optimal solutions such that the cardinality of their intersection is equal to zero, i.e., there is no common element in all optimal solutions. Similar conclusions can be made from Theorem 12 and Corollary 9 if we replace each optimal solution by its complement to the ground set.

One of the major problems, when solving NP-hard problems by means of the branch-and-bound approach, is the choice of the branching element which keeps the search tree as small as possible. Using tolerances we are able to ease this choice. Namely, if there is an element from the optimal solution of the current relaxed NPhard problem (we assume that this optimal solution is a non-feasible solution to the original NP-hard problem) with a positive upper tolerance, then this element is in all optimal solutions of the current relaxed NP-hard problem. Hence, branching on this element means that we enter a common part in all possible search trees emanating from each particular optimal solution of the current relaxed NP-hard problem. Therefore, branching on an element with a positive upper tolerance is not only necessary for finding a feasible solution to the original NP-hard problem but also is a best possible choice. An interesting direction of research is to develop tolerance based $b-n-b$ type algorithms for different NP-hard problems with the objective functions of types $\sum$ and $\Pi$.

Many modern heuristics for finding high quality solutions to a NP-hard problem delete high cost elements and save the low cost ones from a relaxed NPhard problem. A drawback of this strategy is that in terms of either high or low cost elements the structure of all optimal solutions to a relaxed NP-hard problem cannot be described. A tolerance of an element is the cost of excluding or including that element from the solution at hand. Hence, another direction of research is to develop tolerance based heuristics for different NPhard problems with the objective functions of types $\Sigma$ and $\Pi$.

\section{ACKNOWLEDGEMENT}

The research of all authors was supported by a DFG grant SI 657/5, Germany and SOM Research Institute, University of Groningen, the Netherlands.

This article is dedicated to the former project leader, Prof. Dr. Jop Sibeyn, who is missed since a snow-hike in spring 2005. He was involved in the application of the DFG project SI $657 / 5$ and has contributed to the results presented here by lively and inspiring discussions.

\section{REFERENCES}

1. Sotskov, Y.N., V.K. Leontev and E.N. Gordeev, 1995. Some concepts of stability analysis in combinatorial optimization. Discrete Appl. Math., 58: 169-190. 
2. Greenberg, H.J., 1998. An Annotated Bibliography for Post-solution Analysis in Mixed Integer and Combinatorial Optimization. Woodruff, D.L. (Ed.), Advances in Computational and Stochastic Optimization, Logic Programming and Heuristic Search. Kluwer Academic Publishers, pp: 97-148.

3. Gal, T. and H.J. Greenberg, (Eds.), 1997. Advances in Sensitivity Analysis and Parametric Programming. Internat. Ser. Oper. Res. Management Sci. 6. Kluwer Academic Publishers, Boston.

4. Reinfeld, N.V. and W.R. Vogel, 1958. Mathematical Programming. Prentice-Hall, Englewood Cliffs, N.J.

5. Murty, K.G., 1968. An algorithm for ranking all the assignments in order of increasing cost. Oper. Res., 16: 682-687.

6. Van der Poort, E.S., M. Libura, G. Sierksma and J.A.A. Van der Veen, 1999. Solving the k-best traveling salesman problem. Comput. Oper. Res., 26: 409-425.

7. Balas, E. and M.J. Saltzman, 1991. An algorithm for the three-index assignment problem. Oper. Res., 39: 150-161.

8. Goldengorin, B. and G. Jäger, 2005. How to make a greedy heuristic for the asymmetric traveling salesman competitive. SOM Research Report 05A11, University of Groningen, The Netherlands. (http://som.eldoc.ub.rug.nl/reports/themeA/2005/0 5A11/).

9. Goldengorin, B., G. Jäger and P. Molitor, 2006. Tolerance based contract-or-patch heuristic for the asymmetric TSP. Third Workshop on Combinatorial and Algorithmic Aspects of Networking, Chester, United Kingdom, July 2, T. Erlebach (Ed.). Lecture Notes in Comput. Sci.

10. Goldengorin, B., G. Sierksma and M. Turkensteen, 2004. Tolerance Based Algorithms for the ATSP. Graph-Theoretic Concepts in Computer Science. 30th Intl. Workshop, WG 2004, Bad Honnef, Germany, June 21-23, Hromkovic, J., Nagl, M., Westfechtel, B. (Eds.). Lecture Notes in Comput. Sci., 3353: 222-234.

11. Turkensteen, M., D. Ghosh, B. Goldengorin and G. Sierksma, 2005. Tolerance-Based Branch and Bound Algorithms. A EURO Conf. for Young OR Researchers and Practitioners, ORP3, Valencia, Spain, Sep. 6-10, Maroto, C. et al. (Eds.). ESMAP, S.L., pp: 171-182.

12. Helsgaun, K., 2000. An effective implementation of the Lin-Kernighan traveling salesman heuristic. Eur. J. Oper. Res., 126: 106-130.

13. Chin, F. and D. Hock, 1978. Algorithms for updating minimal spanning trees. J. Comput. System Sci., 16: 333-344.
14. Gordeev, E.N., V.K. Leontev and I.K. Sigal, 1983. Computational algorithms for finding the radius of stability in selection problems. USSR Comput. Math. Math. Phys., 23: 973-979.

15. Gusfield, D., 1983. A note on arc tolerances in sparse minimum-path and network flow problems. Networks, 13: 191-196.

16. Kravchenko, S.A., Y.N. Sotskov and F. Werner, 1995. Optimal schedules with infinitely large stability radius. Optimization, 33: 271-280.

17. Libura, M., 1991. Sensitivity analysis for minimum hamiltonian path and traveling salesman problems. Discrete Appl. Math., 30: 197-211.

18. Ramaswamy, R. and N. Chakravarti, 1995. Complexity of determining exact tolerances for min-sum and min-max combinatorial optimization problems. Working Paper WPS-247/95, Indian Institute of Management, Calcutta, India, pp: 34.

19. Shier, D.R. and C. Witzgall, 1980. Arc tolerances in minimum-path and network flow problems. Networks, 10: 277-291.

20. Sotskov, Y.N., 1993. The stability of the approximate boolean minimization of a linear form. USSR Comput. Math. Math. Phys., 33: 699707.

21. Tarjan, R.E., 1982. Sensitivity analysis of minimum spanning trees and shortest path trees. Inform. Process. Lett., 14: 30-33.

22. Volgenant, A., 2006. An addendum on sensitivity analysis of the optimal assignment. Eur. J. Oper. Res., 169: 338-339.

23. Ramaswamy, R., J.B. Orlin and N. Chakravarti, 2005. Sensitivity analysis for shortest path problems and maximum capacity path problems in undirected graphs. Math. Program., Ser. A, 102: 355-369.

24. Gal, T., 1995. Sensitivity Analysis, Parametric Programming, and Related Topics: Degeneracy, Multicriteria Decision Making, Redundancy. W. de Gruyter, Berlin and New York.

25. Goldengorin, B. and G. Sierksma, 2003. Combinatorial optimization tolerances calculated in linear time. SOM Research Report 03A30, University of Groningen, The Netherlands, (http://som.eldoc.ub.rug.nl/reports/themeA/2003/ 03A30/).

26. Hall, N.G. and M.E. Posner, 2004. Sensitivity analysis for scheduling problems. J. Scheduling, 7: 49-83.

27. Bang-Jensen, J. and G. Gutin, 2002. Digraphs: Theory, Algorithms and Applications. Springer, London.

28. Van Hoesel, S. and A. Wagelmans, 1999. On the complexity of postoptimality analysis of $0 / 1$ programs. Discrete Appl. Math., 91: 251-263. 